\title{
UICN
}

\section{Biodiversité et carbone organique des sols : entretenir les zones arides}

Peter Laban, Graciela Metternicht et Jonathan Davies

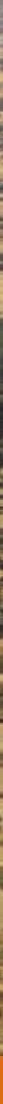

UICN initiative mondiale pour les iones arides 


\section{À propos de l'UICN}

L'UICN est une Union de Membres composée uniquement gouvernements et d'organisations de la société civile. II fournit aux organisations publiques, privées et non gouvernementales les connaissances et les outils nécessaires pour le progrès humain, le développement économique et la conservation de la nature.

Créée en 1948, I'UICN est aujourd'hui le réseau environnemental le plus important et le plus diversifié au monde, exploitant les connaissances, les ressources et l'apport de plus de 1300 organisations membres et quelque 10000 experts. C'est l'un des principaux fournisseurs de données d'évaluations et d'analyses de la conservation. La multiplicité de ses membres permet à I'UICN de jouer le rôle d'incubateur et de référentiel fiable en matière de meilleures pratiques, d'outils et de normes internationales.

L'UICN offre un espace neutre où diverses parties prenantes, y compris les gouvernements, les ONG, les scientifiques, les entreprises, les communautés locales, les organisations de peuples autochtones et autres peuvent travailler ensemble pour forger et mettre en œuvre des solutions pour relever les défis environnementaux.

En collaboration avec de nombreux partenaires et sympathisants, I'UICN met en œuvre un grand portefeuille diversifié de projets de conservation dans le monde entier. Combinant les dernières percées scientifiques avec les connaissances traditionnelles des communautés locales, ces projets visent à inverser la perte d'habitat, à restaurer les écosystèmes et à améliorer le bien-être des populations.

www.UICN.org

https://twitter.com/UICN/

\section{À propos de l'AFD}

L'AFD est la banque française de développement. Publique et solidaire, elle s'engage sur des projets qui améliorent concrètement le quotidien des populations, dans les pays en développement, émergents et l'Outremer. Intervenant dans de nombreux secteurs - énergie, santé, biodiversité, eau, numérique, formation-, l'AFD accompagne la transition vers un monde plus sûr, plus juste et plus durable, un monde en commun. Son action s'inscrit pleinement dans le cadre des objectifs de développement durable (ODD). Présente dans 108 pays via un réseau de 85 agences, l'AFD finance, suit et accompagne aujourd'hui plus de 2500projets de développement. En 2017, l'AFD a consacré 10,34 milliards d'euros au financement de projets dans les pays en développement et en faveur des Outre-mer. 


\section{Biodiversité et carbone organique des sols : entretenir les zones arides}

Peter Labana, Graciela Metternichta,b et Jonathan Davies ${ }^{c}$

aGroupe d'experts des écosystèmes des zones arides, Commission Gestion des Ecosystèmes de l'UICN 'École des sciences biologiques, de la terre et de l'environnement, Centre de recherche PANGEA, Université de Nouvelle-Galles du Sud, Sydney, Australie

'UICN, Union internationale pour la conservation de la nature 
La désignation d'entités géographiques dans ce livre ainsi que la présentation des documents, n'impliquent en aucun cas l'expression de quelque nature que ce soit de l'opinion de l'UICN sur le statut légal d'aucun pays, territoire ou zone ni en ce qui concerne leurs autorités, ni en ce qui concerne leurs frontières et limites.

Les opinions exprimées dans cette publication ne reflètent pas nécessairement celles de l'UICN.

L'UICN rejette toute responsabilité en cas d'erreurs ou d'omissions intervenues lors de la traduction en français de ce document dont la version originale est en anglais. En cas de divergences, veuillez-vous référer à l'édition originale. Titre de l'édition originale : Soil Biodiversity and Soil Organic Carbon: keeping drylands alive. (2018). Gland, Suisse : UICN

Cette publication a été possible en partie grâce au financement de l'Agence Française de Développement.

Publié par: $\quad$ UICN, Gland, Suisse

Droit d'auteur: $\quad$ C 2018 UICN, Union internationale pour la conservation de la nature et de ses ressources

La reproduction de cette publication à des fins non commerciales, notamment éducatives, est permise sans autorisation écrite préalable du [des] détenteur[s] des droits d'auteur à condition que la source soit dûment citée.

La reproduction de cette publication à des fins commerciales, notamment en vue de la vente, est interdite sans autorisation écrite préalable du détenteur des droits d'auteur.

Citation : $\quad$ Peter Laban, Graciela Metternicht, and Jonathan Davies, 2018. Biodiversité et carbone organique des sols : Entretenir les zones arides. Gland, Suisse : UICN. viii $+24 p$

ISBN :

978-2-8317-1890-3 (PDF)

DOI :

https://doi.org/10.2305/IUCN.CH.2018.03.fr

Photo de couverture : Tigray. @ Dr Jonathan Davies, UICN

Mise en page par: $\quad$ Gordon Arara (Unité des publications de l'UICN, Nairobi)

Disponible auprès de : IUCN (International Union

for Conservation of Nature)

Initiative Mondiale pour les Zones arides

Rue Mauverney 28

1196 Gland

Suisse

Tel +41229990000

Fax +41229990002

www.iucn.org/resources/publications

Le texte de ce livre a été imprimé sur Avalon Matt Art 133 gsm. 


\section{Table des matières}

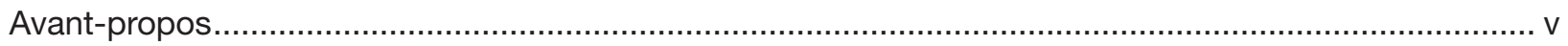

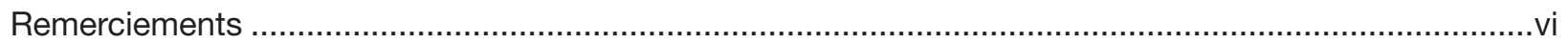

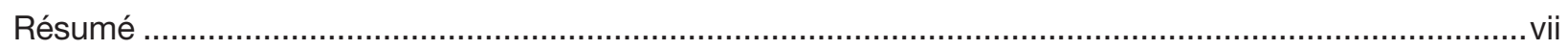

1. Biodiversité des sols, matière organique du sol et carbone organique des sols : introduction et aperçu ....... 1

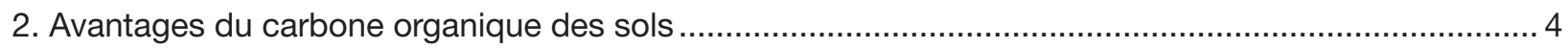

3. Stocks et cycles mondiaux du carbone organique des sols........................................................ 6

4. L'importance du carbone organique des sols dans les zones arides ............................................. 7

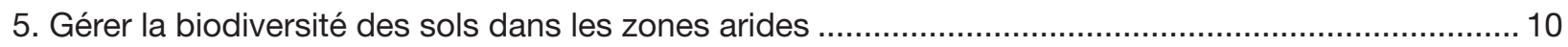

6. Investir dans la biodiversité des sols et le carbone organique des sols ..........................................13

7. Options politiques pour conserver la biodiversité des sols et le carbone organique des sols ............. 16

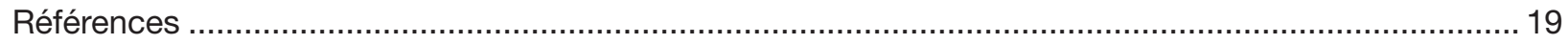

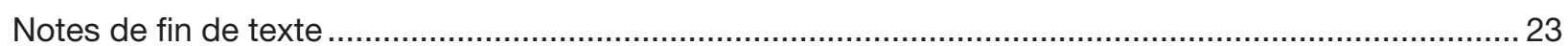




\section{Liste des figures}

Figure 1. Biodiversité des sols et carbone organique des sols ...................................................... 1

Figure 2 Les sols et leurs services et bénéfices écosystémiques..................................................... 5

Figure 3. La vaste étendue des zones arides dans le monde est importante pour le stock total du COS accumulé. a) Systèmes de zones arides et leur répartition spatiale (Source : MEA) ;

(b) Teneur en COS à $1 \mathrm{~m}$ de profondeur en tonnes par hectare (Batjes 2016).

\section{Liste des encadrés}

Encadré 1. Rôle de la faune du sol dans le cycle des nutriments et de l'eau dans les zones arides ....... 2

Encadré 2. Services écosystémiques soutenus par une augmentation du carbone organique des sols....... 4

Encadré 3. Avantages multiples de la réalisation des objectifs de développement durable.................... 4

Encadré 4. Investir dans le COS pour la résilience climatique - Australie............................................... 6

Encadré 5. Permanence du carbone organique des sols des zones arides........................................... 7

Encadré 6. Investir dans le COS et les prairies pour l'élevage et la sécurité en eau - Asie de l'Ouest.. 11

Encadré 7. Augmenter le COS et améliorer les rendements ........................................................... 12

Encadré 8. Les incitations à investir dans la GDT sont souvent autres que financières .......................13

Encadré 9. Investir dans le COS et l'agroforesterie pour la production alimentaire - Afrique ................ 13

Encadré 10. Restauration de paysages à grande échelle en Chine........................................................ 14

Encadré 11. Financer les avantages externes de la gestion agricole durable des zones arides en Australie ...... 14

Encadré 12. Cas d'investissements durables dans les pâturages au Portugal ....................................... 15

Encadré 13. Investissements durables dans les parcours en Jordanie ................................................. 15 


\section{Avant-propos}

Le sol est une fine membrane pourtant vitale qui couvre la surface non submergée de notre planète. II gère les cycles du carbone, de l'azote et de l'eau dont dépend la vie sur terre et abrite une immense diversité d'espèces, qui contribuent toutes aux services précieux et vitaux de la société.

Mais le sol est aussi fragile qu'il est abondant. La croissance de la population humaine entraîne une demande accrue de nourriture, de fibres et de carburant, qui à son tour exerce une pression insoutenable sur les sols de la planète. Au point que, aujourd'hui, jusqu'à 33\% des terres sont soit modérément ou fortement dégradées en raison de l'érosion, de la salinisation, du compactage, de l'acidification et des contaminants chimiques et autres des sols.

La santé des sols, en trouvant leur place au sein des objectifs de développement durable notamment sous la cible 15.3 (Neutralité en matière de dégradation des sols) et autres objectifs mondiaux fixés par la Convention des Nations Unies sur la lutte contre la désertification, attire enfin l'attention nécessaire dans les discussions nationales et mondiales.

Biodiversité et carbone organique des sols : entretenir les zones arides renforce le discours sur la gestion des sols et renforce le message sous-jacent selon lequel le sol est un bien public qui nécessite une évaluation économique et des dispositions institutionnelles connexes visant à le protéger pour le bien-être général de la société. Cette note technique présente un certain nombre de considérations opérationnelles, tirant parti des expériences pratiques et des leçons tirées à travers le monde, et démontre qu'une multitude d'acquis en matière de développement pourraient être obtenus grâce à la gestion durable des terres.

Nous sommes convaincus que cette note technique constituera une ressource inestimable pour les praticiens et les décideurs qui cherchent à passer de la théorie à la gestion pratique de nos sols.

Reconnaissant qu'il reste encore un long chemin à parcourir, et comme nous le soulignons dans cette note technique, nous estimons que nous sommes aujourd'hui en position de force pour transformer la conscience mondiale en action et travailler collectivement pour arrêter le déclin de cette ressource magique qui engendre la vie.

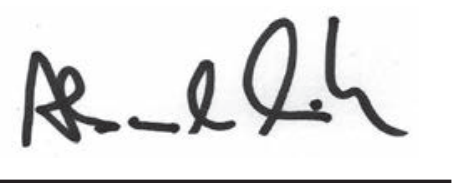

Alexander Müller Directeur général TMG - Töpfer Müller Gaßner Thinktank pour la durabilité Berlin, Allemagne

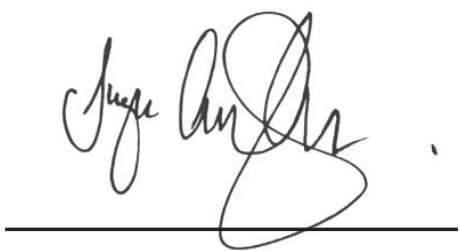

Inger Andersen Directrice générale UICN IUCN 


\section{Remerciements}

Ce document s'est appuyé sur un rapport de Cameron Allen, intitulé « Report on Soil Organic Carbon and Soil Biodiversity in Drylands ${ }^{1}$ » (juillet 2017). Le travail a été renforcé par les contributions de Claire Ogali, Guyo Roba et Razingrim Ouedraogo de l'UICN.

Le rapport a été soumis à une révision par les pairs, notamment Alexander Mueller et le Dr Martial Bernoux. Kirk Olsen, Patrick Gonzales et Jonathan O'Donnell de la Commission mondiale des aires protégées de l'UICN et lan Hannam de la Commission mondiale de l'environnement de l'UICN y ont également contribué à travers des évaluations externes.

Le rapport a été rendu possible grâce au soutien l'Agence Française de Développement. 


\section{Résumé}

D'ici à 2050, la population mondiale devrait atteindre 9 milliards et le monde se devra produire $60 \%$ de nourriture en plus, tandis que 1,8 milliard de personnes vivront dans des zones déficitaires en eau. Alors que la production alimentaire augmente à l'échelle mondiale, les terres dont dépend l'agriculture se dégradent à un rythme alarmant, compromettant les progrès futurs. Les terres dégradées produisent moins de nourriture et stockent moins d'eau et de carbone, ce qui aggrave l'insécurité alimentaire et hydrique et contribue aux changements climatiques.

La biodiversité des sols et le carbone organique des sols sont essentiels au fonctionnement des écosystèmes et déterminent en grande partie le rôle de la terre dans la production de nourriture, le stockage de l'eau et l'atténuation des changements climatiques. Ils sont la clé pour débloquer les multiples avantages économiques et environnementaux - la multifonctionnalité - de la terre.

À l'échelle mondiale, on estime que la contribution de la biodiversité des sols en termes de valeur des services écosystémiques varie entre 1,5 et 13 billions de dollars américains par an. Pourtant, malgré son importance mondiale, la biodiversité des sols est souvent négligée dans les politiques publiques et est en train de disparaître à un rythme considérable due aux méthodes de gestion des terres non viables, l'érosion des sols et d'autres processus de dégradation des sols. Selon les estimations, entre un quart et un tiers des terres dans le monde sont dégradées, ce qui entraîne la baisse de la production agricole, la perturbation de cycles hydrologiques et la libération de gaz à effet de serre séquestrés.

\section{Impacts de la dégradation des terres}

\section{Atténuation du changement climatique}

- L'on estime que les stocks mondiaux de carbone organique des sols (COS) sont supérieurs au carbone de l'atmosphère et de la végétation terrestre combiné. Lorsque le sol est érodé, le COS est redistribué et une partie est perdue

\section{Production alimentaire}

- La dégradation des terres et les changements climatiques pourraient réduire les rendements agricoles et entraîner une baisse de $25 \%$ de la production alimentaire.

\section{Réserve en eau}

- Le sol stocke les deux tiers de l'eau douce de la planète et ce rôle est déterminé par le niveau de matière organique dans le sol.

Les sols des zones arides apportent une contribution importante - environ un tiers - aux stocks mondiaux de biodiversité des sols et de carbone organique des sols, et ils peuvent fortement contribuer à la production alimentaire mondiale puis à l'atténuation du changement climatique. Ils représentent $42 \%$ des terres du monde, soit $44 \%$ de toutes les terres cultivées et produit $50 \%$ du cheptel mondial. Les zones arides sont particulièrement utiles pour le stockage du carbone en raison de leur degré élevé de permanence - la durée pendant laquelle le carbone est stocké dans le sol - par rapport aux zones humides.

L'étendue de la dégradation des terres dans les zones arides est similaire à celle mondiale, toutefois les solutions peuvent différer de celles qui conviendraient aux zones humides. Le niveau relativement élevé de pauvreté et de sous-développement dans les zones arides signifie que les facteurs de dégradation sont différents et que la nature des politiques et des investissements pour lutter contre la désertification devrait différer en conséquence.

La restauration ou la préservation de la biodiversité des sols et du carbone organique des sols nécessite soit une augmentation des apports en matière organique, soit une réduction des pertes de carbone, ou les deux. II est particulièrement important de préserver le carbone organique des sols (COS), voire l'augmenter si possible. Dans les sols des zones arides, les niveaux du COS sont souvent bas et proches du point de basculement où la restauration ne serait plus possible, laissant place à une dégradation irréversible des sols. Cependant, les mesures visant à accroître la biodiversité des sols et le carbone organique des sols peuvent prendre de nombreuses années. La restauration des terres dégradées pouvant être coûteuse, il est préférable a priori d'éviter de sa dégradation et ce par l'adoption de méthodes de gestion durable des terres et la protection durable de paysages agricoles.

On sait que de nombreuses pratiques agricoles, tournant essentiellement autour de la gestion intégrée de l'eau et des nutriments du sol, des mesures de contrôle de l'érosion et de la conservation de la couverture végétale augmentent la biodiversité des sols et le carbone organique des sols. Les pratiques agricoles durables qui ont été largement utilisées dans les zones arides comprennent l'agroforesterie, l'agriculture de conservation et le pastoralisme. Cependant, l'adoption ou le maintien de ces pratiques est faible dans 
les zones arides les plus pauvres du monde, où la croissance démographique et la demande pour une plus grande productivité agricole et la sécurité en matière d'eau sont des plus élevées. Ces régions seront confrontées à la dégradation des terres à moins que la gestion durable des terres ne devienne un élément central des programmes de développement agricole.

La gestion durable des terres exige souvent des investissements en main-d'œuvre et en énergie et nécessite de nouvelles compétences, connaissances, équipements et intrants. Des politiques et des investissements innovants sont donc nécessaires pour encourager son adoption par les exploitants de la terre. Une partie importante de la solution consiste à récompenser ou à encourager la gestion durable des terres, ou " multifonctionnalité ", à grande échelle en exposant les multiples avantages y relatifs plutôt que de maximiser les biens ou services individuels. Cela inclut les valeurs perçues comme externalités par la société dans son ensemble.

Le Programme de développement durable à l'horizon 2030 accroît la demande sur les sols pour assurer la sécurité alimentaire, hydrique et énergétique, la protection de la biodiversité et l'atténuation des changements climatiques, renforçant ainsi la place centrale qu'occupe les sols dans les politiques mondiales de l'environnement et du développement. La Cible 15.3 sur la Neutralité en matière de dégradation des sols, reflète la prise de conscience croissante que la terre, et par extension la biodiversité des sols et du carbone organique des sols, est à la fois une ressource naturelle et un bien public qui sous-tend un développement durable plus large.

Pour une bonne gouvernance de nos ressources foncières communes, les gouvernements devraient viser à protéger et à promouvoir la multifonctionnalité des terres : pour s'assurer que les utilisateurs des terres utilisent des approches durables qui sont mesurées par rapport à la fourniture des multiples biens et services. Atteindre cet objectif nécessite un certain nombre de mesures prioritaires qui sont :

1. Évaluer la gestion des terres par rapport à la fourniture durable des multiples biens et services ;

2. S'appuyer sur les politiques et la législation pour favoriser l'intensification de la gestion durable des terres et la restauration ou la réhabilitation des paysages ;

3. Améliorer les mécanismes de gouvernance locale qui soutiennent les utilisateurs des terres dans les méthodes de gestion durable des terres ;

4. Renforcer l'information sur le foncier afin de soutenir la planification et le suivi à l'échelle du paysage ;

5. Établir des services de vulgarisation efficaces qui aident les utilisateurs des terres à adopter des méthodes de gestion durable des terres ;

6. Créer des conditions favorables à l'investissement privé dans la gestion durable des terres. 


\section{Biodiversité des sols, matière organique des sols et carbone organique des sols: introduction et aperçu}

Les sols sont parmi les habitats les plus riches en espèces sur terre, abritant une abondance d'espèces qui permettent aux sols de fonctionner et de se développer. Beaucoup de ces espèces aident essentielles à la diversité fonctionnelle et à la résilience du sol et des écosystèmes qui dépendent du sol. La biodiversité des sols est un indicateur de la qualité du sol : une plus grande diversité des espèces entraîne une plus grande stabilité du sol en termes de capacité à remplir des fonctions clés telles que le recyclage des nutriments, l'assimilation des déchets organiques et la conservation de la structure du sol'1.

La biodiversité des sols, le carbone organique des sols et la matière organique du sol sont étroitement liés mais distincts (Figure 1). La biodiversité des sols rreflète le mélange d'organismes vivants dans le sol, y compris les bactéries, les champignons, les protozoaires, les insectes, les vers et autres invertébrés et les vertébrés. Ces organismes interagissent les uns avec les autres, ainsi qu'avec les plantes et les petits animaux, formant une toile d'activité biologique ${ }^{2}$. La plupart des espèces vivent dans la couche supérieure du sol - entre 2 et $3 \mathrm{~cm}$ - où la concentration de matière organique et de racines est la plus élevée. Les croûtes biologiques du sol, par exemple, sont des communautés de mousses, de lichens et de micro-organismes à la surface du sol qui conservent particulièrement la fertilité du sol dans les zones arides du monde entier ${ }^{3}$.

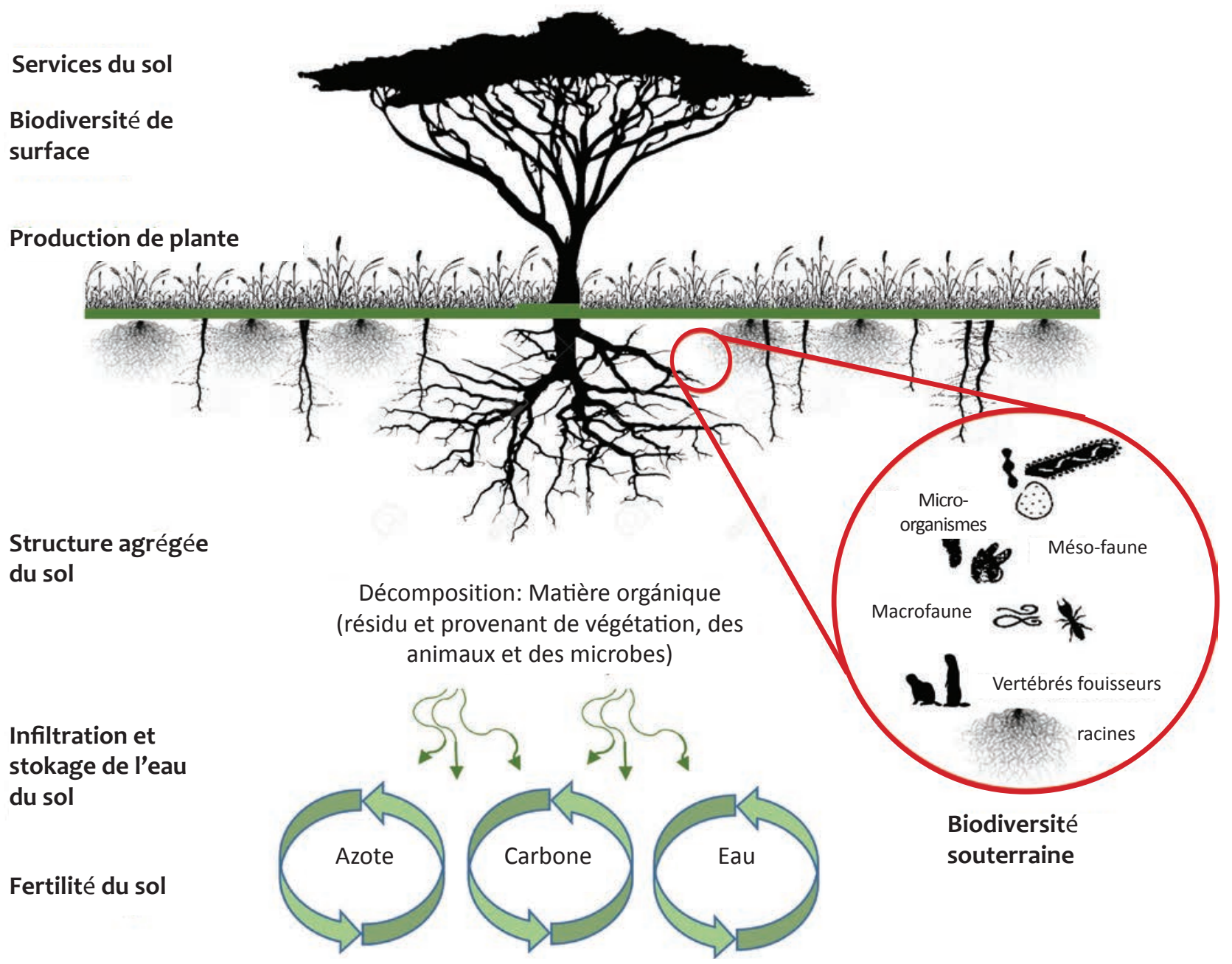

Figure 1. Biodiversité des sols et carbone organique des sols 
Au sens large, la matière organique du sol (MOS) englobe tous les composants organiques d'un sol, y compris la biomasse vivante (tissus végétaux et animaux intacts et micro-organismes), les racines mortes et autres résidus végétaux, les tissus morts et l'humus du sol. La MOS et les débris de tissus morts laissés par les plantes et les animaux (détritus) sont essentiels pour l'activité biologique dans le sol et constituent la principale source d'énergie, de nutriments et d'habitat pour la grande majorité des organismes du sol. Le carbone organique des sols représente environ 50 à $60 \%$ de la matière organique du sol. Une estimation de la MOS est habituellement faite par la mesure du COS , avec l'application d'un facteur de 1,9 à 2 pour convertir le COS en MOS4.

Dans les zones arides, telles que le Sahel, le Moyen-Orient ou l'Australie, la plus grande partie de la biodiversité se trouve en dessous de la surface du sol et sa conservation est cruciale pour la sécurité alimentaire et hydrique, comme l'illustre l'Encadré 1.

\section{Encadré 1. Rôle de la faune du sol dans le cycle des nutriments et de l'eau dans les zones arides}

Les insectes sont importants pour le cycle des nutriments et de l'eau dans les zones arides. Par exemple, les bousiers, dans les familles d'insectes Scarabaeidae et Geotrupidae, jouent un rôle majeur dans la productivité de nombreuses prairies. Ces coléoptères enrichissent les éléments nutritifs du sol, aèrent le sol et améliorent la porosité et le drainage du sol en enterrant les excréments des animaux. Les termites jouent également un rôle crucial, en particulier dans les savanes d'Afrique, d'Australie et d'Amérique du Sud, en modifiant les propriétés du sol et en créant des « îlots de fertilité » qui favorisent la croissance des plantes. Les termitières augmentent l'hétérogénéité dans le paysage et jouent un rôle majeur dans le cycle des nutriments et l'infiltration de l'eau 5 . Cela contribue à la résilience des écosystèmes, aide les écosystèmes des zones arides à se remettre de la sécheresse et les stabilise face aux changements climatiques.

Dans les écosystèmes arides et semi-arides, on rencontre fréquemment une terre avec une croûte biologique du sol constituée de communautés d'organismes vivants qui vivent sur le sol, ou à quelques millimètres de la surface du sol. Cette croûte est une association entre la cyanobactérie, les algues, les champignons microscopiques et autres espèces, et les particules du sol. Les croûtes biologiques du sol jouent un certain nombre de rôles écologiques, notamment la fixation du carbone et de l'azote et la stabilisation du sol. Ils affectent également l'infiltration d'eau et la germination des graines ainsi que d'autres propriétés qui peuvent avoir des implications positives ou négatives pour la productivité des terres et la croissance de la végétation. Les croûtes biologiques du sol sont plus enclines à se constituer lorsque la couverture végétale est réduite, étant donné que la cyanobactérie a besoin de l'énergie solaire pour la photosynthèse. Ils peuvent donc être un symptôme de la dégradation des terres, mais ils peuvent aussi stabiliser les sols et freiner la dégradation ${ }^{6}$.

La biodiversité des sols contribue au développement de la végétation de surface en décomposant les résidus végétaux, un processus qui convertit les nutriments organiquement conservés en formes organiques et minérales contribuant au renouvellement des plantes (p. ex. azote) $)^{7}$. La biodiversité des sols augmente la résilience des sols, en termes de capacité à « rebondir » vers une santé fonctionnelle après une perturbation grave. La contribution de la biodiversité des sols aux services écosystémiques au niveau mondial a été estimé entre 1,5 et 13 billions USD par an ${ }^{8}$.

Les sols sont des écosystèmes complexes et les organismes du sol et les composants minéraux interagissent pour générer une diversité d'une complexité élevée. La composition minérale du sol et les organismes du sol déterminent ensemble la structure du sol et sa fertilité. Elle dépend des facteurs de formation du sol tels que le matériau d'origine, le relief, le climat, l'hydrologie, les organismes biologiques et le temps. La capacité du sol à retenir l'humidité est dans une large mesure déterminée par la MOS/COS et la structure du sol. L'eau qui est stockée dans les sols sert de source à $90 \%$ de la production agricole mondiale et représente environ $65 \%$ de l'eau douce mondiale ${ }^{9}$. En effet, une étude a indiqué que chaque perte de $1 \mathrm{~g}$ de MOS diminue la teneur en humidité disponible du sol de 1 à $10 \mathrm{~g}^{10}$. La productivité végétale (pour la productivité agricole et la biodiversité) dépend donc directement des organismes du sol, qui régulent la disponibilité et l'absorption des nutriments, maintiennent la structure du sol et régulent les processus hydrologiques ${ }^{11}$.

La quantité et la qualité de la MOS et du COS sont donc des facteurs majeurs de biodiversité dans le sol, fournissant une source d'énergie et de nourriture pour les micro-organismes qui sont essentiels aux processus biologiques se déroulant dans le sol. À son tour, la biodiversité des sols contribue à la formation de la MOS par la décomposition et la production d'humus. Ces nombreuses interactions sont différentes et complexes et créent de multiples boucles de rétroaction de renforcement biologique, chimique et physique. 
Les sols à haute teneur en matière organique sont capables de supporter une plus grande diversité végétale, ce qui a pour effet d'augmenter la MOS et le COS, tout en améliorant la biodiversité des sols. Bien que peu d'études aient quantifié ces effets ${ }^{12}$, des recherches récentes ont utilisé le COS comme proxy de la MOS et de la densité apparente du sol, démontrant que même des réductions marginales de la teneur en COS de l'ordre de $1 \%$ peuvent avoir un impact négatif significatif sur les services naturels du sol et des écosystèmes ${ }^{13}$.

L'importance du carbone organique des sols pour des résultats positifs en matière d'environnement et de développement a été reconnue à la suite des décisions éloquentes prises à la douzième session de la Conférence des Parties (COP) de la Convention des Nations Unies sur la lutte contre la désertification en octobre 2015. Le carbone organique des sols est reconnu comme un déterminant majeur de la productivité agricole et de la sécurité en eau, et constitue la pierre angulaire de la résilience de la biodiversité et du changement climatique. Cette importance est reflétée dans la cible 15.3 des objectifs de développement durable pour parvenir à un monde neutre en termes de dégradation des sols, notamment par l'un de ses trois indicateurs : «évolution des stocks de carbone de surface et dans le sol ». Cependant, il y a une faible convergence entre les nombreuses branches de la science, de la pratique et de la politique qui traitent du carbone organique des sols et de sa relation avec la biodiversité. En conséquence, les multiples avantages du carbone organique des sols sont facilement ignorés et risquent d'être perdus.

Cette note technique a été développé pour combler le manque de connaissance de la relation entre la biodiversité des sols et le carbone organique des sols, en particulier dans les zones arides, et le rôle de la biodiversité des sols dans la fourniture de services écosystémiques. Le note technique est rédigé pour les conseillers techniques des ministères responsables de l'environnement, de l'agriculture et des ressources naturelles. Cela englobe des scientifiques, des conseillers en politiques et des groupes de la société civile, dont beaucoup connaissent le vaste domaine, mais ne seraient pas au courant des dernières recherches sur la biodiversité des sols et le carbone organique des sols.

La note technique s'appuie sur deux précédentes notes techniques de I'UICN sur les parcours et sur la neutralité ${ }^{14}$. dans la dégradation des terres. Il synthétise les principales recherches sur la biodiversité des sols des zones arides et fournit des exemples à l'appui dans de brèves études de cas, avec des liens pour de plus amples informations. Des leçons sont tirées de cette information et les principales recommandations qui émergent sont fournies dans la section finale. Les informations compilées dans ce rapport sont tirées d'un document d'information plus long intitulé "Carbone organique des sols et biodiversité des sols dans les zones arides" écrit par Cameron Allen et attend d'être publié sur le site web de l'UICN. 


\section{Avantages du carbone organique des sols}

Les avantages du carbone du sol, et par extension la biodiversité des sols, sont largement décrits dans la littérature. L'augmentation du COS améliore la qualité et la fertilité du sol, améliore la productivité du sol, la croissance de la végétation et favorise l'accumulation de carbone dans le sol. L'augmentation de la quantité et de la qualité du carbone organique des sols améliore la stabilité de la structure du sol, la capacité de rétention de l'eau, la porosité et la fertilité du sol ${ }^{15}$. contribuant ainsi à amélioration d'une large gamme de services écosystémiques (Encadré 2 et Figure 2).

\section{Encadré 2. Services écosystémiques soutenus par une augmentation du carbone organique des sols}

1. Augmentation des rendements des cultures et augmentation de la production alimentaire (jusqu'à 2,3 milliards de tonnes de production agricole supplémentaire par an, soit 1,4 billion de dollars US) ${ }^{16}$

2. Augmentation de la capacité de rétention d'eau du sol, infiltration et stockage d'eau

3. Une plus grande biodiversité de surface et souterraine (la contribution mondiale de la biodiversité des sols aux services écosystémiques est estimée entre 1,5 et 13 billions USD par an) ${ }^{17}$

4. Stockage du carbone et régulation du climat (au moins la moitié des réductions d'émissions nécessaires pour atteindre l'objectif de 2 degrés convenu par la communauté internationale pourrait provenir des secteurs fonciers des principaux pays émetteurs qui possèdent une partie substantielle des zones arides) ${ }^{18}$

La valeur de la biodiversité des sols n'est souvent appréciée que lorsque celle-ci commence à décliner. La perte de carbone organique des sols, en particulier lorsque les niveaux initiaux sont faibles, comme c'est le cas dans les régions arides, entraîne invariablement la dégradation des terres et de leurs fonctions écosystémiques. Selon les estimations, entre un quart et un tiers des terres dans le monde sont affectées par une forme de dégradation des sols ${ }^{19}$, qui contribue à la baisse de la production agricole, à la perturbation des cycles de l'eau, à la libération de gaz à effet de serre et à de nombreux autres coûts pour la société. Ces impacts négatifs doivent être envisagés dans une perspective où la population humaine nécessiterait $60 \%$ de nourriture supplémentaire d'ici à 2050 et d'ici à 2025, 1,8 milliard de personnes vivront dans des zones où la pénurie d'eau est absolue. La dégradation des terres et les changements climatiques pourraient réduire les rendements agricoles et entraîner une baisse de $25 \%$ de la production alimentaire ${ }^{20}$.

La biodiversité des sols et le carbone organique des sols sont donc une base importante d'un large éventail de services écosystémiques dans les quatre catégories de services écosystémiques standard (voir Figure 2). Elle est donc essentielle à la multifonctionnalité d'un paysage, et la raison pour laquelle le renforcement des investissements et de la législation en matière de gestion durable des terres est considérée comme essentielle à la réalisation de nombreux des objectifs de développement durable (voir Encadré 3). La cible 15.3 des ODD vise à atteindre un monde neutre en matière de dégradation des sols d'ici à 2030 , en maintenant et en augmentant la quantité de ressources foncières saines et productives.

\section{Encadré 3. Avantages multiples de la réalisation des objectifs de développement durable}

La gestion durable des terres peut contribuer simultanément à plusieurs ODD, notamment l'éradication de la pauvreté (objectif 1), la sécurité alimentaire et hydrique (objectifs 2 et 6), la protection de la biodiversité (objectif 15) et l'atténuation et l'adaptation au changement climatique (objectif 13). Par exemple, la recherche a estimé que restaurer seulement $12 \%$ des terres agricoles dégradées pourrait augmenter les revenus des petits exploitants de 35 à 40 milliards de dollars par an et aider à nourrir 200 millions de personnes chaque année en 15 ans, tout en augmentant la résilience aux sécheresses, aux pénuries d'eau, puis en réduisant les émissions de GES ${ }^{21}$. 


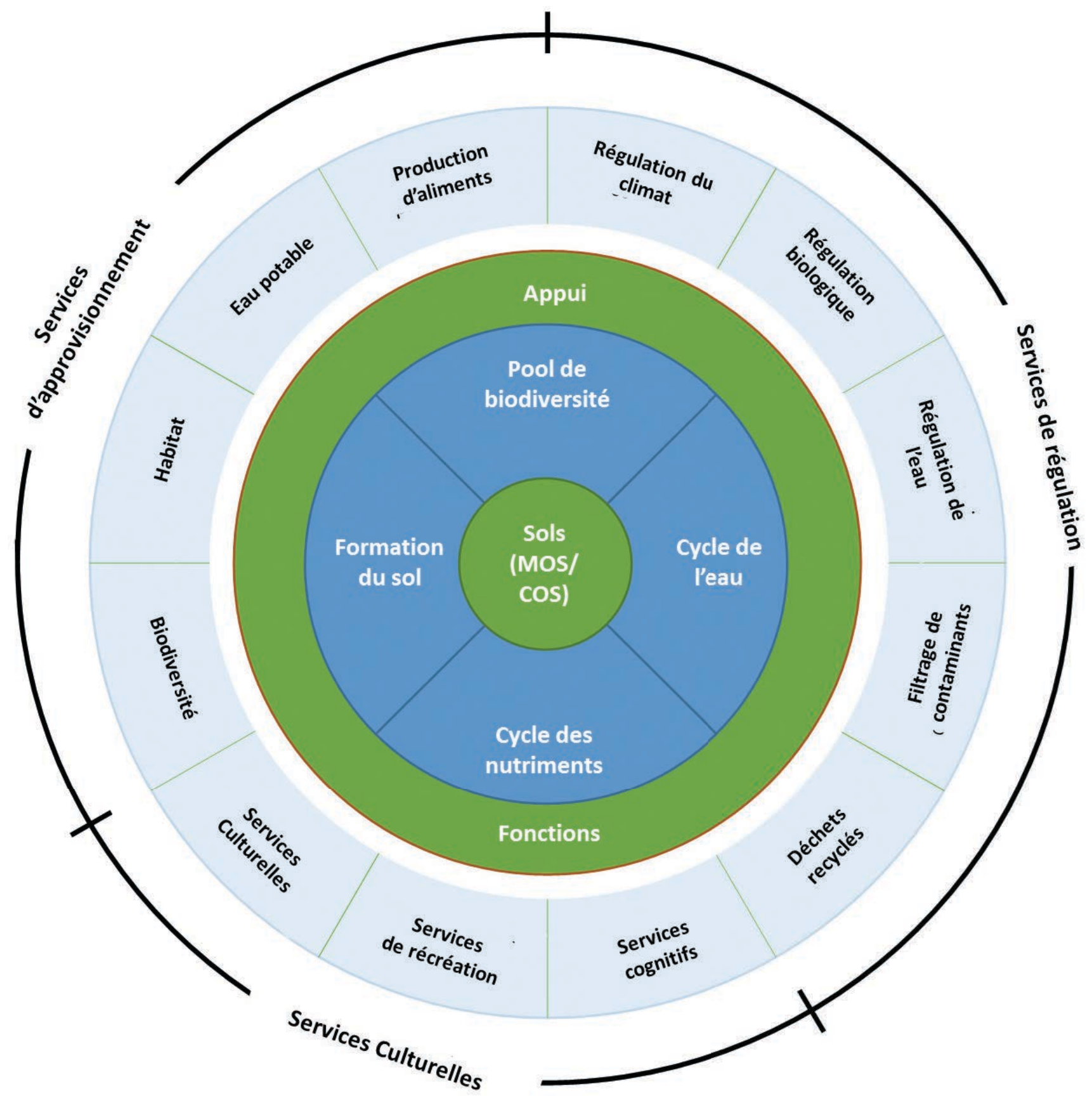

Figure 2 Les sols et leurs services et bénéfices écosystémiques 


\section{Stocks et cycles mondiaux du carbone organique des sols}

Le COS fait partie du cycle mondial de carbone très important. Le réservoir du COS n'est pas statique et il fait constamment le tour des différents réservoirs de carbone du sol, de la végétation, de l'océan et de l'atmosphère. Le sol est le deuxième plus grand stock de carbone après les océans et représente le tiers du stock mondial de carbone ${ }^{22}$. En dépit des variations observables entre les estimations, les stocks mondiaux du COS ont été estimés à environ 1500 GtC dans le premier mètre du sol, soit supérieur à celui de l'atmosphère (800 Gt C) et la végétation terrestre (500 Gt C) ${ }^{23}$.

L'ampleur spatiale et temporelle de stockage du COS est très variable et est déterminée par différents facteurs abiotiques et biotiques, y compris le type de sol, l'utilisation de la terre et les conditions climatiques ${ }^{24}$. Les stocks du COS les plus importants sont situés dans des zones telles que les zones humides et les tourbières, dont la plupart se trouvent dans des régions de pergélisol et sous les tropiques ${ }^{25}$.

Le carbone organique dans les sols est un continuum qui peut être divisé conceptuellement en différents réservoirs. La plupart des approches considèrent trois réservoirs qui sont fonction de leur stabilité physique et chimique : réservoirs rapides (réservoirs labiles ou actifs avec un renouvellement de 1-2 ans) ; réservoirs intermédiaires (réservoirs partiellement stabilisés avec un renouvellement de 10 à 100 ans) ; et réservoirs lents (réservoirs réfractaires ou stables avec un renouvellement très lent de 100-1000 ans) ${ }^{26}$. La partie rapide ou labile, partie vitale du COS, est considérée comme importante pour influencer la santé du sol et la séquestration du SOC, tandis que la fraction lente ou stable du COS contribue à la capacité de rétention des nutriments du sol et à la séquestration du carbone à long terme ${ }^{27}$.

Les niveaux du COS reflètent un équilibre dynamique entre les apports de carbone organique frais et les émissions de carbone rejetées dans l'atmosphère, et de carbone organique dissous et particulaire entrant dans les eaux de surface ou souterraines. L'érosion des sols entraîne la redistribution du COS à l'échelle de la localité, du paysage et de la région. La restauration ou la préservation du COS dans les sols nécessite une augmentation des apports de carbone organique ou une réduction des pertes, ou encore les deux. Cependant, les effets de tels changements peuvent prendre des années à se manifester, les effets de l'utilisation des terres et des méthodes de gestion influant sur les tendances des niveaux du COS depuis des décennies après l'introduction de nouvelles pratiques ${ }^{29}$.

\section{Encadré 4. Investir dans le COS pour la résilience climatique - Australie}

Dans le sud-ouest de l'Australie-Occidentale, la valeur marginale du COS dans les systèmes de culture (valeur d'un sol avec $1 \mathrm{t}$ C/ha de plus par rapport à un sol standard) a été estimée entre 5,6 et 6,9 \$ US/ t C/ha/an, en fonction de la zone de la pluviométrie et du type de culture. Environ $75 \%$ de cette valeur agronomique est la valeur estimée de la séquestration du carbone, $20 \%$ est la valeur de remplacement de l'azote et $5 \%$ est la valeur estimée de l'amélioration de la productivité. Valeur ( $1 \$ \mathrm{AU}=$ environ 0 , $75 \$$ US) $)^{28}$. 


\section{L'importance du carbone organique des sols dans les zones arides}

Les zones arides englobent une grande diversité biologique et certains des systèmes d'utilisation des terres les plus importants au monde. Elles entretiennent plus de 2 milliards de personnes et $25 \%$ des espèces menacées dans le monde ${ }^{30}$. À l'échelle mondiale, les écosystèmes des zones arides couvrent environ $42 \%$ de la surface de la Terre, y compris certains des écosystèmes les plus menacés au monde ${ }^{31}$ (Figure 3 ). Ils représentent $44 \%$ des terres cultivées dans le monde et produit $50 \%$ du cheptel mondial ${ }^{32}$, aet contiennent une variété d'habitats importants pour les espèces végétales, les arbres fruitiers et les micro-organismes ${ }^{33}$. Les zones arides abritent de nombreux habitats biologiquement et culturellement diversifiés de la planète, avec des niveaux élevés d'endémisme des espèces et du patrimoine naturel ${ }^{34}$.

Les zones arides sont des régions tropicales et tempérées avec un indice d'aridité ${ }^{2}$ de moins de 0,65 , où les précipitations moyennes sont inférieures aux pertes d'humidité potentielles dues à l'évaporation et à la transpiration ${ }^{35}$. À l'aide de l'indice d'aridité, on peut distinguer quatre catégories de zones arides : les zones arides subhumides, semi-arides, arides et hyperarides. Alors que les terres cultivées ne se trouvent généralement que dans les zones les moins arides, les parcours eux représentent environ les trois quarts des zones arides. Les parcours comprennent des biomes dominés par l'herbe, mais contiennent également une quantité considérable de biomasse ligneuse, en particulier dans les zones subhumides et semi-arides ${ }^{36}$. Environ $72 \%$ des zones arides se trouvent dans les pays en développement et cette proportion augmente en même temps que l'aridité : presque toutes les terres hyperarides se trouvent dans les pays en développement ${ }^{37}$.

À première vue, l'importance mondiale des zones arides pour le stockage du carbone organique des sols peut ne pas être apparente. La rareté de l'eau dans les zones arides limite la productivité des plantes, ce qui affecte l'accumulation de MOS et du COS dans les sols. En conséquence, les sols des zones arides ont généralement une faible teneur en carbone organique, souvent inférieure à $1 \%$ de la masse du sol. Dans les sols des prairies ou des forêts, le COS peut atteindre 4-5\%, mais le labour du sol agit pour libérer le carbone stocké ; dans les zones tempérées le COS est d'environ 1-2\% dans les sols cultivés.

Due à leur grande étendue, le stock total du COS accumulé dans les zones arides est important, représentant environ $30 \%$ des stocks globaux totaux de SOC, soit environ $450 \mathrm{GtC}$, soit environ autant que le carbone organique stocké dans toute la végétation terrestre ${ }^{38}$. De plus, le temps de résidence du carbone dans les sols des zones arides est prolongé en raison de leur aridité et dure beaucoup plus que dans les autres sols ${ }^{39}$ (Encadré 5). L'étendue spatiale des zones arides, combinée à la superficie importante affectée par la dégradation des terres, signifie que les zones arides joueront un rôle crucial dans l'effort mondial de séquestration du carbone atmosphérique et de réduction de l'ampleur du changement climatique anthropique.

Le potentiel de séquestration du carbone dans les sols est lié à la saturation du carbone du sol (ou capacité maximale de stabilisation du carbone), qui est le point auquel d'autres apports de carbone dans le sol n'augmenteront plus le stock de carbone du sol ${ }^{41}$. L'état conditionnel des sols des zones arides serait très inférieur à leur point de saturation maximal de carbone, en raison principalement de leur aridité et donc de

\section{Encadré 5. Permanence du carbone organique des sols des zones arides}

La permanence fait référence à la longévité du stock de carbone, c'est-à-dire la durée pendant laquelle le COS accru reste dans le sol. Le COS oscille constamment entre le réservoir rapide, avec une faible permanence, mais avec la plus grande influence sur la santé du sol, et le réservoir lent, qui a la plus grande permanence et la plus grande capacité de rétention des nutriments. Les moyens par lesquels les stocks de carbone du sol sont augmentés, tels que la fumure ou le boisement, peuvent affecter le taux de perte de carbone du sol qui en résulte, et donc la permanence du stock. Cependant, les différences peuvent être minimes par rapport à la perte qui peut résulter de changements extrêmes dans l'utilisation ou la gestion des terres ${ }^{40}$. 

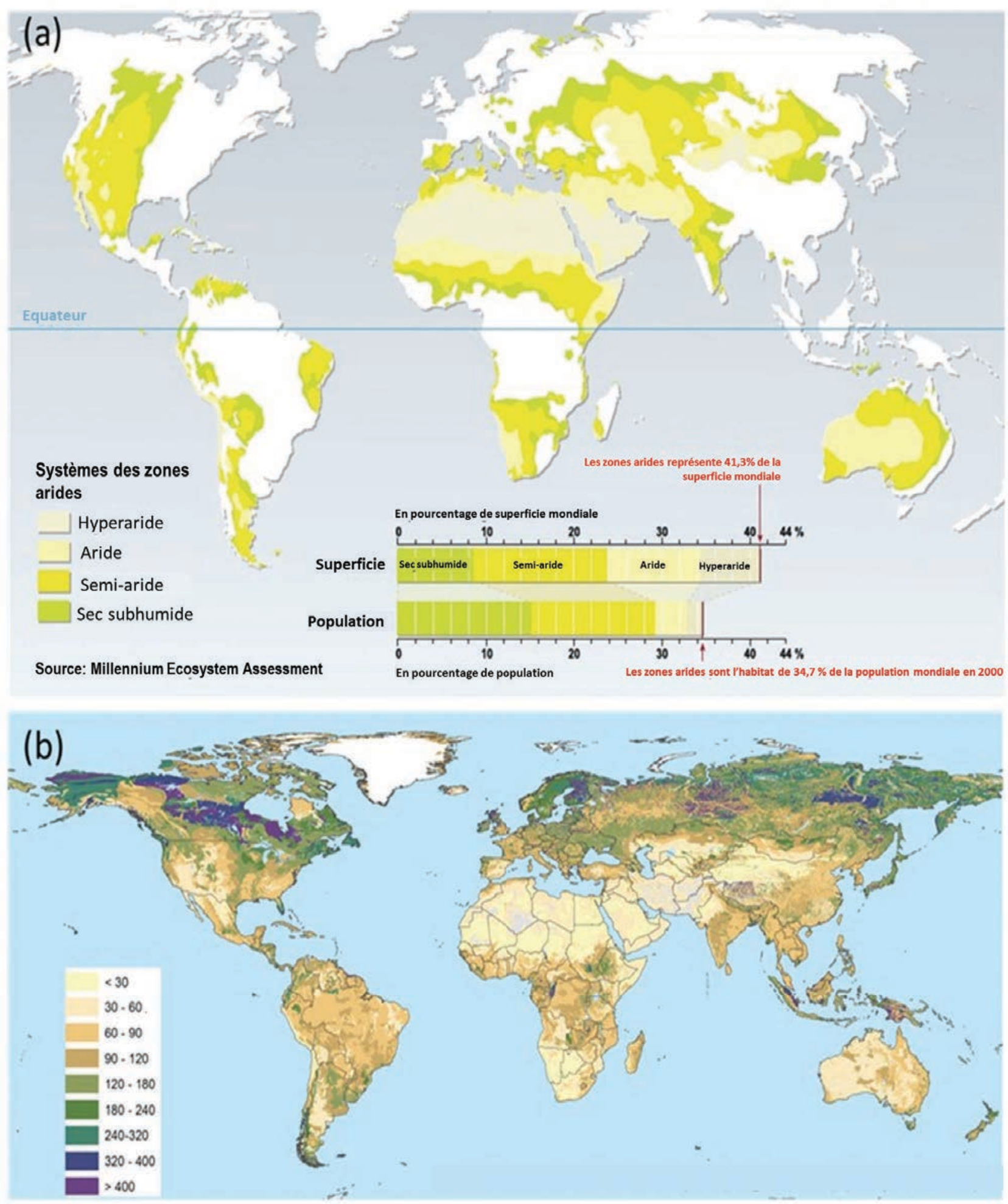

Figure 3. La vaste étendue des zones arides dans le monde est importante pour le stock total du COS accumulé. a) Systèmes de zones arides et leur répartition spatiale (Source : MEA); (b) Teneur en COS à $1 \mathrm{~m}$ de profondeur en tonnes par hectare (Batjes 2016). 
leur productivité globale relativement faible. De plus, étant donné que la dégradation des terres affecte entre un quart et un tiers des zones arides, de grandes superficies sont susceptibles d'avoir des niveaux du COS encore plus bas.

La biodiversité des sols et le carbone organique des sols peuvent être restaurés par l'application de matière organique ou par la réduction des pertes de carbone, ou encore les deux. Cela est urgent dans les sols dégradés des zones arides, où le taux de carbone est souvent bas et proches du point de basculement, où la restauration n'est plus possible et où la dégradation des terres risque de devenir irréversible. Cependant, les mesures visant à accroître la biodiversité des sols et le carbone organique des sols peuvent prendre de nombreuses années. La restauration des terres dégradées peut également être coûteuse et il est préférable de l'éviter a priori, par l'adoption de méthodes de gestion durable des terres et la protection de paysages agricoles ou pastoraux gérés de manière durable.

La qualité et la productivité du sol des zones arides peuvent être améliorées en renforçant la teneur en matière organique du sol (d'où les niveaux du COS) à travers d'autres méthodes de gestion comme l'agriculture sans labour ${ }^{42}$ et la gestion durable des prairies. La recherche sur les sites de prairies protégés par la communauté en Jordanie a démontré le potentiel d'augmentation de la production de biomasse sur les terres dégradées ${ }^{43}$. Sauf mauvaise gestion de la prairie, de nombreuses études ont montré que la prairie a un effet positif sur le stock de carbone du sol dans les zones arides ${ }^{44}$.

Si les sols des zones arides sont naturellement bien en deçà de leur point de saturation maximal en carbone, rehausser leurs niveaux de COS au-delà de l'état naturel peut nécessiter une modification substantielle des conditions naturelles et des caractéristiques de l'écosystème ; par exemple à travers l'irrigation et le boisement. De telles approches ont été proposées comme solutions pour séquestrer le carbone atmosphérique, bien qu'il faille mieux comprendre les risques potentiels à la permanence (en raison de l'augmentation de l'humidité du sol) ainsi que les impacts possibles sur la biodiversité des zones arides.

Les prairies tropicales, qui représentent une grande partie des zones arides, semblent avoir un potentiel de séquestration de carbone plus élevé qu'on ne le pensait ${ }^{45}$. avec un estimation qu'elles stockent globalement environ $343 \mathrm{Gt} \mathrm{C}$ : près de $50 \%$ de plus que ce qui est stocké dans les forêts du monde entier ${ }^{46}$. Les prairies d'Afrique, par exemple, pourraient séquestrer entre 0,007 et $0,042 \mathrm{Gt} C$ année- $1^{47}$. La conversion des prairies en cultures annuelles peut entraîner une perte de $60 \%$ des stocks de carbone du sol et une perte de $95 \%$ du carbone de surface ${ }^{48}$.

Les zones arides possèdent un fort potentiel de séquestration du carbone et certaines organisations se réfèrent aux zones arides comme des " points lumineux » de séquestration de carbone. Bien qu'il reste des interrogations sur les meilleures pratiques pour chaque site, les zones arides doivent être considérées comme des zones clés pour les investissements dans la gestion durable des stocks de carbone. De tels investissements doivent être activement gérés pour restaurer, préserver et augmenter leurs niveaux de carbone organique et favoriser leur potentiel de séquestration ${ }^{49}$. 


\section{Gérer la biodiversité des sols dans les zones arides}

L'Agenda 2030 accroît la demande en matière de sécurité alimentaire, hydrique et énergétique, protège la biodiversité et atténue les changements climatiques. Cela renforce le rôle central des sols dans les politiques mondiales de l'environnement et du développement. En conséquence, cela a conduit à la notion de " sécurité du sol " ou de "santé du sol ». La sécurité du sol se concentre sur la conservation et l'amélioration des ressources du sol du monde pour produire des aliments, des fibres et de l'eau douce, contribue à l'énergie et la durabilité climatique et garanti la biodiversité et la protection globale de l'écosystème ${ }^{50}$.

Le COS peut être considéré comme un indicateur universel de la sécurité ou de la santé du sol. La réduction en COS va également réduire la sécurité/santé du sol ainsi que les avantages qui auraient pu en découler. Les facteurs qui influencent la séquestration du COS dans les sols comprennent le climat, le type de sol, la couverture végétale et les méthodes de gestion. Dans les zones arides, le rôle et les avantages de la MOS et du COS sont particulièrement liés à leurs propriétés physiques, biologiques et chimiques ${ }^{51}$.

II existe différents moyens de garantir la durabilité de la gestion des terres ou des écosystèmes. Ceux-ci peuvent être catégorisés soit comme des actions directes d'utilisation des terres (mesures entreprises par les utilisateurs des terres) soit comme des mesures habilitantes (mesures prises par les « facilitateurs » pour promouvoir ou améliorer l'adoption d'actions directes d'utilisation des terres). Une variété de techniques de gestion des terres peut préserver ou augmenter le carbone organique des sols, qui nécessite principalement une gestion efficace de la matière organique et de l'eau afin de garantir un niveau de fertilité suffisant pour une production alimentaire durable ${ }^{52}$. Beaucoup de ces pratiques offrent de multiples avantages, tel que l'augmentation de la productivité alimentaire, des ressources en eau et de la biodiversité, ainsi que l'atténuation et l'adaptation au changement climatique.

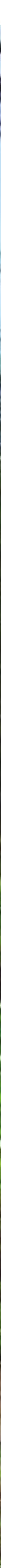




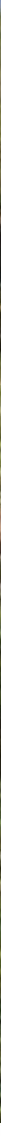

La plupart des bonnes pratiques en matière de gestion du carbone organique des sols évoluent autour de la gestion intégrée de l'eau et des nutriments, des mesures de contrôle de l'érosion et du maintien de la couverture végétale ${ }^{53}$. Par exemple, l'agriculture de conservation assure un labour minimum, le paillage du sol et la rotation des cultures, qui se combinent pour augmenter la matière organique et la fertilité du sol ${ }^{54}$. S. La gestion durable des parcours met l'accent sur la gestion de la durée, du moment et de l'intensité du pâturage afin d'assurer un impact optimal sur les herbivores, comme la dispersion des graines, la fumure et l'élimination de la végétation morte ${ }^{55}$. L'agroforesterie est un système dans lequel des arbres ou des arbustes sont plantés parmi les cultures ou dans les prairies pour augmenter la productivité, la diversité et la résilience des fermes ${ }^{56}$. Ces pratiques appliquent toutes des principes de synergie et de complémentarité afin de conserver la structure du sol, l'humidité du sol et les nutriments ${ }^{57}$ et éventuellement augmenter la capacité de stockage du carbone.

\section{Encadré 6. Investir dans le COS et les prairies pour l'élevage et la sécurité en eau - Asie de l'Ouest}

En Jordanie, la gestion communautaire des parcours est en cours d'extension afin de restaurer et de protéger la terre et la biodiversité. L'analyse géo-spatiale et les études de faisabilité sur la repousse de la végétation sous la protection communautaire indiquent que la restauration de terres de parcours en Jordanie peut être réalisé sur environ $23.470 \mathrm{~km}^{2}$ de terres de parcours, soit environ $30 \%$ de la superficie totale des terres de parcours du pays. Les études montrent également que les investissements dans la gestion durable des parcours (SRM), la conservation des sols et des eaux, l'élevage écologique, les herbes et les plantes médicinales, l'éco-tourisme et les énergies renouvelables peuvent contribuer à l'augmentation de la biomasse et du fourrage, à la recharge des eaux souterraines, à la réduction de l'envasement des réservoirs d'eau, à la séquestration du carbone et à la conservation de la biodiversité. L'évaluation économique indique que la SRM dans ces pâturages augmenterait la production fourragère estimée à environ 9,45 millions $\$$ US, et la recharge des eaux souterraines estimée à environ 11 millions de $\$$ US par $a^{58}$. 
II existe un désaccord considérable sur la façon de protéger et de restaurer le COS des prairies, en particulier en ce qui concerne les impacts positifs et négatifs des herbivores sur l'écologie des prairies. Certains auteurs signalent une augmentation du stock du COS avec des prairies gérées de façon intensive ${ }^{59}$ tandis que d'autres rapportent que le COS peut être augmenté en réduisant ou en éliminant le pâturage ${ }^{60}$. Les niveaux élevés de pâturage permanent étant particulièrement nuisibles, le type de gestion des pâturages est essentiel pour influer le COS. Par exemple, on a constaté que le pâturage en rotation augmente le COS par rapport au pâturage continu de faible intensité ${ }^{61}$. Une bonne gestion des pâturages synchronise généralement le pâturage avec le cycle de croissance des graminées, en exploitant le rôle positif joué par les herbivores dans la sélection et la distribution des espèces palatables, et la modification des propriétés physiques et chimiques du sol ${ }^{62}$. La gestion des pâturages affecte également le cycle de l'azote, puisque les herbivores renvoient de l'azote digestible à des taux de concentration élevés à travers les urines ${ }^{63}$. La gestion du feu et la fertilisation ont également été proposées comme moyens pour accroître la séquestration du carbone dans les prairies, mais dans la pratique, la plus grande partie du potentielle de séquestration dans les prairies non dégradées survient à travers des changements dans les pratiques de pâturage.

Bien que ces approches de gestion des terres aient encore du mal à trouver leur place dans certains pays, il convient également de noter qu'elles ont été largement adoptées dans d'autres. Par exemple, environ 558 millions de personnes dans le monde utilisent des systèmes agro-forestiers sur $43 \%$ (plus d'un milliard d'hectares) de toutes les terres agricoles, dont 320 millions en Amérique du Sud, 190 millions en Afrique subsaharienne et 130 millions en Asie du Sud-Est ${ }^{64}$. L'agroforesterie n'est pas exclusive aux zones arides, mais elle s'avère populaire pour la protection et la restauration des terres dégradées dans un nombre croissant de pays arides.

\section{Encadré 7. Augmenter le COS et améliorer les rendements}

L'adoption de méthodes de gestion durable des terres agricoles et des sols dégradés peut améliorer la qualité du sol, notamment la capacité de rétention d'eau, la capacité d'échange de cations, l'agrégation du sol et la susceptibilité à la croûte terrestre et à l'érosion. Des recherches menées en Argentine, en Inde et dans le Sahel ouest africain ont montré que les rendements des cultures peuvent être augmentés de $20-70 \mathrm{~kg} / \mathrm{ha}$ pour le blé, de $10-50 \mathrm{~kg} / \mathrm{ha}$ pour le riz et de $30-300 \mathrm{~kg} / \mathrm{ha}$ pour le maïs pour chaque $1000 \mathrm{~kg} / \mathrm{ha}$ d'augmentation du réservoir de carbone organique des sols dans la zone racinaire. Cela présente des avantages évidents pour la sécurité alimentaire dans les régions en développement, et pourrait également compenser les émissions de combustibles fossiles à raison de 0,5 GtC/an grâce à la séquestration du carbone dans les sols agricoles ${ }^{65}$. Cependant, atteindre de telles augmentations de COS nécessite des niveaux élevés d'apport en eau, ce qui constitue une contrainte majeure dans la plupart des zones arides et implique des externalités potentielles et des coûts d'opportunité qui n'ont pas encore été suffisamment explorés. 


\section{Investir dans la biodiversité des sols et le carbone organique des sols}

Les solutions pour la gestion durable du sol afin de conserver la biodiversité des sols et le COS sont bien connues et ont été validées dans différents pays et contextes géographiques. Néanmoins, de nombreux pays continuent d'accorder la priorité à des pratiques d'utilisation des terres moins intensives, tandis que les approches durables sont marginales. L'intégration de la GDT présente de nombreux défis, y compris des barrières d'attitude chez les décideurs et les investisseurs et des contraintes de capacités chez les utilisateurs des terres.

La GDT peut être intégrée dans l'aménagement du territoire ${ }^{66}$ et des incitations économiques pour la mise en œuvre de la GDT peuvent être développées à travers des modèles d'entreprise durables, des subventions ou des paiements pour le service écosystémique. Ce dernier nécessite souvent des connaissances, compétences, technologies et ressources appropriées, notamment la main-d'œuvre, l'énergie et des ressources financières. Les coûts d'investissement initiaux peuvent être élevés ${ }^{67}$ et le décalage dans la réalisation des bénéfices de l'investissement peut rendre ces projets peu attractifs. Les retours sur investissement et autres facteurs d'investissement doivent donc être identifiés pour encourager son adoption par les propriétaires terriens (agriculteurs, arboriculteurs, éleveurs).

\section{Encadré 8. Les incitations à investir dans la GDT sont souvent autres que financières}

Une étude menée auprès de 500 agriculteurs en Afrique de l'Ouest a révélé que les principaux facteurs à investir étaient la présence d'enfants (âgés de 6 à 14 ans) dans le ménage, la propriété foncière, le régime foncier, la sensibilisation et la formation aux mesures de conservation et l'accès à d'autres sources de liquidités telles que les envois de fonds ${ }^{70}$ Certains de ces facteurs pourraient être ciblés pour l'extension de l'adoption de pratiques durables dans les zones arides des pays en développement (ou des pays en transition économique). Dans de nombreux cas, cela impliquera également des politiques de gouvernance locale mieux articulées en ce qui concerne les responsabilités foncières et de gestion ${ }^{71}$. Les pratiques de GDT continuent d'être réduits à une minorité d'utilisateurs et de praticiens innovants, mettant en exergue le fait que les défis à l'adoption de la GDT sont plus complexes que le simple coût d'adoption des nouvelles technologies, notamment écologiques, institutionnelles, économiques et socioculturelles ${ }^{72}$.

Les avantages des pratiques de GDT sont appréciés par des personnes autres que les propriétaires terriens (Encadré 8) et ces externalités peuvent être utilisées pour encourager les incitations à l'investissement, par exemple au moyen de paiements pour les services écosystémiques (PSE). Il y a eu des tentatives d'estimation de la valeur économique des services individuels fournis par les sols, le COS et la biodiversité des sols, y compris les études récentes qui ont compilé ces données dans un format plus accessible ${ }^{68}$. TCes estimations approximatives varient toutefois considérablement, allant de 2 à $22.219 \$ / h / a^{69}$. Alors que les estimations soulignent la nécessité de poursuivre les recherches, elles indiquent néanmoins que la valeur économique totale des services écosystémiques du COS est susceptible d'être importante.

\section{Encadré 9. Investir dans le COS et l'agroforesterie pour la production alimentaire - Afrique}

Le bassin versant de la forêt de Kelka, dans la région de Mopti au Mali, couvre une superficie de plus de 300000 ha, dont une grande partie a été dégradée. La restauration des terres dégradées et l'adoption de l'agroforesterie pourraient générer 500 \$ US par hectare et par an sur un horizon de 25 ans, indiquant un rapport bénéfice/coût de 5,2:1 à un taux d'actualisation de $10 \%{ }^{74}$. Des recherches en Europe et en Chine confirment que la composante arbre en agroforesterie a des effets positifs sur la séquestration du carbone, notamment via la capacité des systèmes racinaires plus profonds à stocker le carbone ${ }^{75}$. 
Les retours sur investissement dans la conservation ou l'amélioration des terres peuvent être exprimés audelà des conditions monétaires, par des avantages non monétaires accrus tels qu'un bien-être plus élevé, des moyens de subsistance plus sûrs, une eau moins polluée, des loisirs, un habitat ou une valeur en nature, des expériences spirituelles et esthétiques (Encadré 9). De nombreux utilisateurs des terres, par exemple, sont motivés par les valeurs spirituelles ou culturelles de la terre, et ces services peuvent ne pas être bien mesurés en termes financiers. La seule évaluation économique peut ne pas saisir ce qui est en jeu dans un agenda social et écologique et il y a des risques à se fier exclusivement aux déclencheurs du marché pour atteindre la durabilité ${ }^{73}$. Les retours sur investissement ne sont pas la seule incitation pour amener les gens à investir, et se fonder sur ces motifs financiers suppose que la valeur de la nature et de l'environnement ne dépend que de la fonction qu'il a pour la production et la consommation de l'homme.

\section{Encadré 10. Restauration de paysages à grande échelle en Chine}

En Chine, les projets du plateau de Loess (1994-2005) ont mobilisé un financement de 491 millions de dollars US et freiné l'érosion des sols sur près d'un million d'hectares de terres dégradées. Les projets ont mis l'accent sur l'arrêt des activités qui ont conduit à la dégradation - en particulier la plantation sur les pentes abruptes, la coupe des arbres et le pâturage en plein air des chèvres ; l'équipement lourd introduit pour construire des terrasses plus larges et plus robustes pour la culture des céréales, et encouragé les agriculteurs à planter des arbres et à permettre aux terres marginales de redevenir sauvages. Les projets ont fortement augmenté les rendements céréaliers, restauré le paysage et permis à plus de 2,5 millions de personnes de sortir de la pauvreté en triplant les revenus des agriculteurs. L'érosion du sol a été freinée sur 900000 ha de terres et les pertes de sol ont été réduites de 60 à $100 \mathrm{Mt}$ par an. Le stockage du carbone dans les sols a également augmenté, principalement en raison de la restauration des forêts et des prairies. Le modèle de projet a depuis été étendu à de vastes régions du pays, grâce au programme Chinois «Grain for Green» ${ }^{76}$ d'un montant de 40 milliards de dollars US.

Dans les zones arides, comme dans d'autres écosystèmes, des niveaux plus élevés du COS sont un déterminant clé de la régulation et de l'approvisionnement des services écosystémiques tels que la biodiversité, les flux hydrologiques, la production alimentaire et la séquestration du carbone. Nourrir et investir pour maintenir la qualité et les flux de ces services écosystémiques permet de maintenir des niveaux élevés du COS (Encadré 10). Le défi consiste à mobiliser des ressources financières pour des investissements appropriés en faveur de la multifonctionnalité foncière.

\section{Encadré 11. Financer les avantages externes de la gestion agricole durable des zones arides en Australie}

En Australie, le gouvernement fédéral a élaboré des initiatives visant à encourager les agriculteurs à réduire leurs émissions grâce à leurs méthodes de gestion. La politique principale est un mécanisme basé sur le marché pour payer les agriculteurs à partir d'un fonds de réduction des émissions en utilisant des méthodologies spécifiées dans le cadre de l'Australian Carbon Farming Initiative. L'Initiative permet aux agriculteurs et aux exploitants agricoles de gagner des crédits de carbone en stockant du carbone ou en réduisant les émissions de GES sur leurs terres. Ces crédits peuvent ensuite être vendus aux personnes et aux entreprises souhaitant compenser leurs émissions. L'adoption des pratiques d'agriculture de conservation dans le secteur australien des céréales en zones arides montre qu'il est possible de réduire les émissions de l'ordre de trois millions de tonnes de CO2 par an. Le principal moteur du changement en Australie semble être la poursuite des avantages liés à la productivité agricole plutôt que les avantages environnementaux liés à la réduction des émissions de GES, soulignant la nécessité de politiques et de méthodes de gestion durable des terres qui procurent un rendement financier aux propriétaires. Les principales pratiques qui sont encouragées par le cadre politique sont les pratiques d'agriculture de conservation telles que l'agriculture sans labour, le maintien du chaume des cultures et la plantation de légumineuses, comme détaillé dans le Carbon Farming Initiative Handbook ${ }^{77}$.

Les prairies qui dominent les zones arides sont parmi les biomes les moins protégés de la planète. La protection de ces zones à travers la promotion d'un pastoralisme durable ou à travers d'autres formes de gestion des pâturages peut empêcher l'augmentation des émissions de carbone, tandis que la restauration des prairies dans les anciennes terres cultivées peut augmenter la séquestration du carbone ${ }^{78}$. Un moyen rentable de sauvegarder la biodiversité des sols et le carbone organique des sols est de protéger contre les labours les terres incultes, telles les prairies naturelles et les forêts. La couverture des aires protégées dans les zones arides est proche de la moyenne mondiale, c'est-à-dire environ $9 \%$ du territoire est protégé, en deçà de l'aspiration de l'Objectif d'Aichi 11 (" au moins 17 pour cent de la surface terrestre et des eaux intérieures. . . seront protégés à travers. . . des systèmes d'aires protégées et d'autres mesures de conservation efficaces selon les zones »"79). 
De nombreuses zones arides offrent un large espace d'innovation en développant de multiples chaînes de valeur qui privilégient la multifonctionnalité. Cela a été réalisé, par exemple, à travers la commercialisation de produits naturels récoltés de manière durable, l'éco-tourisme et la certification de produits durables. Bien que chaque option ait ses limites, elles peuvent fournir des incitations importantes pour une gestion durable associée à la possibilité de diversification économique et de croissance. Le soutien politique au développement des petites et moyennes entreprises peut être particulièrement pertinent pour remplir ce créneau.

\section{Encadré 12. Cas d'investissements durables dans les pâturages au Portugal}

Le Portugal a mis en place un système de compensation du carbone basé sur l'amélioration des pâturages des zones arides, allouant 8,5 millions d'euros à quelque 400 agriculteurs participants à l'amélioration d'environ 42000 ha de prairies dans le but de séquestrer 0,91 million de tonnes d'équivalent de dioxyde de carbone. Pour y parvenir, les agriculteurs utilisent une technique connue sous le nom de SBPPRL ${ }^{3}$, une biodiversité des prairies permanentes ensemencées riches en légumineuses, ce qui augmente le carbone dans les sols dégradés. Les prairies ont généralement un stock de carbone faible et il a été démontré que la technique augmente considérablement le COS dans ces sols dégradés. L'amélioration de 42000 ha permettrait aux agriculteurs de gagner entre 150 à 200 euros par hectare de pâturages planté ${ }^{80}$. Les modalités de financement de la restauration et de la gestion durable du carbone organique des sols des zones arides pourraient s'inspirer des systèmes de paiement pour les services écosystémiques (PSE) (voir Encadré 12). Une justification du PSE pourrait être utilisée pour expliquer les flux financiers internationaux provenant de bénéficiaires externes vers ceux qui mettent en œuvre et contribuent aux investissements dans la gestion durable des terres, et donc le maintien et l'augmentation du $\operatorname{COS}^{81}$. Des investissements habilitants sont également nécessaires dans de nombreux pays, par exemple pour sécuriser le régime foncier de manière à atténuer les conséquences inéquitables, ou pour mettre en place une infrastructure permettant le développement de la chaîne de valeur.

Des mécanismes de compensation par les bénéficiaires hors site au profit des propriétaires terriens seront nécessaire pour assurer le flux des bénéfices étant donné que les avantages liés à la conservation ou au renforcement des services écosystémiques des zones arides s'étendent au-delà des utilisateurs directs de la terre. Aussi faudra-t-il des méthodes pour s'assurer que les bénéficiaires « en aval » contribuent aux avantages dont ils bénéficient, c'est-à-dire contribuer à une meilleure recharge et à la disponibilité des eaux souterraines, à la réduction de l'envasement des réservoirs, à une meilleure biodiversité ou meilleure séquestration du carbone. En effet, la société à travers le monde bénéficie également de certains services écosystémiques des zones arides. Ainsi d'autres innovations sont nécessaires pour s'assurer que ces bénéficiaires paient les propriétaires terriens qui grâce à leurs pratiques de méthodes de gestion des terres ${ }^{83}$.

\section{Encadré 13. Investissements durables dans les parcours en Jordanie}

Les programmes d'investissement proposés pour la gestion durable des parcours en Jordanie, en association avec la protection communautaire des parcours et mécanismes de gouvernance locale, ont un fort potentiel de création d'avantages écosystémiques locaux et sociétaux et contribuent globalement à la conservation de la biodiversité, à l'atténuation des changements climatiques et à d'autres objectifs. La contribution de 20 millions USD/an, telle qu'elle est engendrée par les programmes d'investissement proposés dans 30\% des parcours de la Jordanie, pourrait constituer une base pour attirer les capitaux et les flux financiers nécessaires. En effet, des propositions ont été élaborées pour un système de paiement des services écosystémiques qui pourrait comprendre deux paiements de services écosystémiques : un crédit vert pour les pâturages sous forme de subvention aux coopératives d'éleveurs responsables de la gestion durable des parcours dans les zones désignées et un crédit vert pour l'eau aux mêmes coopératives pour améliorer la recharge des eaux souterraines. Un système mondial de paiement pour les services écosystémiques pourrait fournir la plate-forme nécessaire, tandis que les investissements dans l'éco-tourisme et l'énergie renouvelable pourraient fournir les moyens, par le biais de la fiscalité et des licences, aux modalités du PSE au niveau national. Les deux modalités de PSE pourraient fournir les flux de financement (nationaux et internationaux) nécessaires pour investir dans la production de biomasse et la biodiversité, la conservation des sols, l'amélioration des flux en eau, la séquestration du carbone et dans les structures de gouvernance locale requises. Le gouvernement jordanien envisage une série de mesures habilitantes, notamment la création d'une Zone de parcours économique et écologique, de Zones de conservation vérifiées et d'un Fonds de gestion des écosystèmes des parcours ${ }^{82}$.

3 Sown Biodiverse Permanent Pastures Rich in Legumes 


\section{Options politiques pour conserver la biodiversité des sols et le carbone organique des sols}

Cette note technique souligne comment le carbone organique des sols et la biodiversité des sols constituent la base des services écosystémiques terrestres. Ils sont parmi les principaux déterminants de la production alimentaire, de l'approvisionnement en eau, de la biodiversité de surface et de la régulation du climat, et ils jouent un rôle majeur dans les moyens de subsistance ruraux et la réduction des risques de catastrophe. La biodiversité des sols et le carbone organique des sols sont à la fois des ressources naturelles et un bien public, et le gouvernement a un rôle important à jouer pour s'assurer que les terres sont gérées pour le bien-être de la société

Les zones arides offrent plus de possibilités de séquestration du carbone et d'atténuation des changements climatiques que ce qui est largement reconnu. Elles recouvrent une grande partie de la terre et une bonne proportion a perdu son COS dans le processus de dégradation des sols. La restauration et la réhabilitation des zones arides offrent des opportunités importantes à l'échelle mondiale pour la séquestration du carbone. Cependant, les approches conventionnelles de la restauration doivent souvent être adaptées aux conditions locales des zones arides, tandis que les politiques et les investissements doivent être soutenus par de meilleures données sur les niveaux du COS existants et potentiels.

Des stratégies gouvernementales sont nécessaires pour orienter les investissements des utilisateurs locaux, des entreprises privées et autres parties prenantes dans les zones arides, afin d'assurer leur responsabilité publique de protection et de promotion de la multifonctionnalité des terres. Les investissements dans la biodiversité des sols et le carbone organique des sols dans les zones arides peuvent générer des bénéfices significatifs en raison du nombre et de la valeur des co-bénéfices, et de la grande superficie des terres concernées. Toutefois, ces investissements doivent être adaptés pour créer de la multifonctionnalité à plus grande échelle plutôt que de ne maximiser que les biens ou les services.

Un certain nombre de mesures peuvent être adoptées pour créer des conditions qui permettent un meilleur investissement dans le carbone organique des sols et la biodiversité des sols. II en ressort six options complémentaires qui sont :

1. Évaluer la gestion des terres par rapport à la livraison durable des multiples biens et services ;

2. S'appuyer sur les politiques et la législation pour permettre l'intensification de la gestion durable des terres et la restauration ou la réhabilitation des paysages ;

3. Améliorer les mécanismes de gouvernance locale qui appuient les utilisateurs des terres dans les méthodes de gestion durable des terres ;

4. Renforcer l'information foncière pour soutenir la planification et le suivi à l'échelle du paysage ;

5. Établir des services de vulgarisation efficaces qui aident les utilisateurs des terres à adopter des méthodes de gestion durable des terres ;

6. Créer des conditions favorables à l'investissement privé dans la gestion durable des terres. 


\section{- Évaluer la gestion des terres par rapport à la livraison durable des multiples biens et services}

Des efforts supplémentaires sont nécessaires pour renforcer les arguments économiques en faveur de la gestion durable du carbone organique des sols et de la biodiversité des sols en fournissant des preuves des multiples avantages économiques et en ayant un regard sur la performance des utilisateurs des terres face aux multiples services écosystémiques. II existe des technologies qui ont été testées dans différents contextes pour démontrer aux utilisateurs des terres en particulier et à la société en général les avantages liés à la gestion durable des terres. Autre cela, les évaluations économiques peuvent guider les politiques, en démontrant le rapport coûts-avantages de l'adoption de la GDT et en identifiant les domaines où des incitations supplémentaires, telles que les paiements pour les services écosystémiques, peuvent être nécessaires pour soutenir la transition vers des pratiques plus durables. Dans certains cas, des preuves supplémentaires sont nécessaires pour déterminer dans quelle mesure le carbone organique des sols et la biodiversité des sols contribuent à la biomasse de surface, à la productivité agricole, au cycle de l'eau et à l'atténuation du changement climatique.

\section{- S'appuyer sur les politiques et la législation pour permettre l'intensification de la gestion durable des terres et la restauration ou la réhabilitation des paysages}

Pour passer des projets périphériques à des investissements de base dans l'agriculture et l'utilisation des terres en matière de la gestion durable des terres, il faut des politiques et des lois. Cela pourrait prendre la forme d'un soutien explicite pour certains types de gestion des terres (agroforesterie, agriculture de conservation, gestion des parcours, par exemple), ou d'une réglementation permettant d'atteindre des indicateurs clés de durabilité, tels que le carbone organique des sols. Des mesures d'encouragement peuvent également être nécessaires lorsque les investissements dans la gestion durable des terres dépassent les moyens financiers ou organisationnels des utilisateurs des terres au niveau local. Dans ce contexte, il est important de comprendre la valeur des stratégies de gestion des risques établies par les utilisateurs des terres car leur réticence à abandonner des stratégies éprouvées ne doit pas être confondue avec l'ignorance. La réalisation de l'utilisation multifonctionnelle des terres nécessite également des accords institutionnels pour la coordination intersectorielle ou la collaboration dans la gestion des terres, et pour les modalités de gouvernance locale en faveur de la gestion des ressources naturelles. Dans l'ensemble, cela nécessite de promouvoir la gestion durable des terres et de hiérarchiser les objectifs nationaux de neutralité en matière de dégradation des sols dans les plan d'action nationaux.

\section{- Améliorer les mécanismes de gouvernance locale qui appuient les utilisateurs des terres dans les méthodes de gestion durable des terres}

Le soutien des pouvoirs publics est également nécessaire pour renforcer la gouvernance locale des ressources naturelles et la sécurité foncière. La sécurisation foncière et une gouvernance locale efficace sur les ressources naturelles sont essentielles pour parvenir à une gestion durable des terres et pour garantir ou augmenter la biodiversité et le carbone organique des sols. Les droits des hommes et des femmes et des peuples autochtones à accéder à la terre et à la gérer doivent être respectés. Cela peut impliquer la mise ou la remise en place d'institutions locales et de règles qui facilitent l'application des connaissances locales; par exemple, les règles locales sur la coupe des arbres, l'accès à l'eau ou le pâturage sur les parcours saisonniers. Le respect des connaissances locales et des institutions, la construction d'un consensus et l'assurance d'une équité par le biais d'approches participatives, de soutien politique, législatif et institutionnel, et la sécurisation des ressources et propriété foncière sont partie intégrante de la gouvernance locale en tant qu'ensemble de systèmes qui contrôlent et assurent la médiation de prise de décision par les acteurs locaux, en consultation avec les parties prenantes externes concernées en matière de développement et de gestion des ressources.

\section{- Renforcer l'information foncière pour soutenir la planification et le suivi à l'échelle du paysage}

Veiller à ce que les principales données biophysiques et socio-économiques relatives à la terre soient disponibles pour les décideurs depuis le niveau local jusqu'au niveau national. Cela comprend des informations sur l'utilisation des terres, le potentiel foncier, le régime foncier, les tendances du changement dans l'utilisation de la terre, la valeur des terres, le potentiel de séquestration du sol et du carbone de surface et l'état de la dégradation des terres. Ces données doivent être à jour et doivent être accessibles à tous les utilisateurs. Dans la plupart des pays, des investissements plus importants sont nécessaires pour le suivi 
de la dégradation des terres, y compris indicateurs convenus par les Parties à la CCNUD pour suivre les progrès réalisés en matière de neutralité dans la dégradation des terres : évolution de la couverture végétale, évolution de la productivité ou du fonctionnement des terres et évolution des stocks de carbone de surface et souterrain.

\section{- Établir des services de vulgarisation efficaces qui aident les utilisateurs des terres à adopter des méthodes de gestion durable des terres}

Les services de vulgarisation doivent être conçus pour faire progresser la gestion durable des terres, ou les éléments clés de la durabilité, comme activité principale. Les gouvernements doivent prendre des décisions sur la manière de promouvoir la gestion durable des terres, car les agriculteurs ont besoin d'une formation sur de nouvelles approches, par exemple en agroforesterie ou en agriculture de conservation. Dans de nombreux cas, cela nécessitera une mise à jour professionnelle importante du personnel de vulgarisation en combinaison avec un investissement dans les programmes de formation professionnelle et d'enseignement supérieur pour surmonter les décennies de promotion de systèmes de gestion des terres non viables. Là où l'adoption de la gestion durable des terres expose les utilisateurs des terres à de nouveaux risques, ou là où les investissements nécessaires sont au-delà de leur capacité financière ou organisationnelle, des incitations et des mesures d'atténuation des risques peut être requises. Ces incitations seront souvent justifiées par la grande valeur et la diversité des bénéfices sociétaux récoltés, depuis le niveau local jusqu'au niveau mondial.

\section{- Créer des conditions favorables à l'investissement privé dans la gestion durable des terres}

La gestion durable des terres nécessite souvent des changements dans les pratiques et les intrants agricoles et, dans certains cas, pourrait également influencer le type de production agricole. Cela a des implications importantes pour le secteur privé. Les entreprises privées ont un rôle majeur à jouer en développant de produits et de services innovants et de nouveaux marchés qui soutiennent les pratiques de GDT. Par exemple, la réduction du travail du sol peut nécessiter des changements dans les régimes d'engrais et d'herbicides et peut nécessiter des machines agricoles adaptées. Une plus grande attention doit être accordée à la diversification des marchés afin de refléter la toute la gamme des valeurs fournies par des terres saines. Cela inclut les marchés pour les services écosystémiques, et pourrait également inclure la certification de produits de niche de grande valeur tels que les herbes médicinales et les produits d'élevage écologiques issus de systèmes agricoles durables ou de paysages. 


\section{References}

Adhikari K. and Hartemink A.E., 2016. Linking soils to ecosystem services-A global review. Geoderma, 262, 101-111. https://doi.org/10.1016/j.geoderma.2015.08.009

Aerni P., 2016. The sustainable provision of environmental services. From regulation to innovation. Springer: Switzerland. https://doi.org/10.1007/978-3-319-19345-8

Álvaro-Fuentes J., López M.V., Cantero-Martinez C. and Arrúe J.L., 2007. Tillage Effects on Soil Organic Carbon Fractions in Mediterranean Dryland Agroecosystems. Soil Science Society of America Journal Abstract - SOIL and WATER MANAGEMENT and CONSERVATION; Vol. 72 No. 2, p. 541-547

Amundson R., Berhe A.A., Hopmans J.W., Olson C., Sztein A.E. and Sparks D.L., 2015. Soil and human security in the 21st century. Science, 348, 1261071. https://doi.org/10.1126/science.1261071

Arriagada R. and Perrings C., 2009. Making Payments for Ecosystem Services Work. Policy Brief, UNEP: Nairobi. 32 pages. Available at: http://bioecon-network.org/pages/UNEP_publications/02\%20PES.pdf

Australian Government, 2012. The Carbon Farming Initiative Handbook, Canberra, Australia, Australian Department of Climate Change and Energy Efficiency.

Bai Z.G., Dent D.L., Olsson L. and Schaepman M.E., 2008. Proxy global assessment of land degradation. Soil use and management, 24, 223-234. https://doi.org/10.1111/j.1475-2743.2008.00169.x

Banwart S., Black H., Cai Z., Gicheru P., Joosten H., Victoria R., Milne E., Noellemeyer E., Pascual U. and Nziguheba G., 2014. Benefits of soil carbon: report on the outcomes of an international scientific committee on problems of the environment rapid assessment workshop. Carbon Management, 5, 185-192. https://doi.org/10.1080/1758 3004.2014 .913380

Bastin J-F., Berrahmouni N., Grainger A., Maniatis D., Mollicone D., Moore R., Patriarca C., Picard N., Sparrow B. and Abraham E. M., 2017. The extent of forest in dryland biomes. Science, 356, 635-638. https://doi.org/10.1126/ science.aam6527

Batjes N., 2016. Harmonized soil property values for broad-scale modelling (WISE30sec) with estimates of global soil carbon stocks. Geoderma, 269, 61-68. https://doi.org/10.1016/j.geoderma.2016.01.034

Batjes N.H., 2004. Estimation of soil carbon gains upon improved management within croplands and grasslands of Africa. Environment, Development and Sustainability 6: 133-143. https://doi.org/10.1023/B:ENVI.0000003633.14591.fd

Baveye P.C., Baveye J. and Gowdy J., 2016. Soil "ecosystem" services and natural capital: Critical appraisal of research on uncertain ground. Frontiers in Environmental Science, 4, 41. https://doi.org/10.3389/fenvs.2016.00041

Beare M., Mcneill S., Curtin D., Parfitt R., Jones H., Dodd M, and Sharp J., 2014. Estimating the organic carbon stabilisation capacity and saturation deficit of soils: a New Zealand case study. Biogeochemistry, 120, 71-87. https://doi. org/10.1007/s10533-014-9982-1

Belnap J. and Lange O.L., 2003. Biological Soil Crusts: Structure, Function, and Management. Ecological Studies, Volume 150. https://doi.org/10.1007/978-3-642-56475-8

Bernoux M. and Chevallier T., 2014. Carbon in dryland soils: multiple essential functions. In: INTERNATIONAL, C. A. (ed.) Les dossiers thematiques du CSFD. France.

Bishop J. and Hill C. (eds.), 2014. Global Biodiversity Finance: The Case for International Payments for Ecosystem Services. Edward Elgar, Cheltenham. https://doi.org/10.4337/9781782546955

Blair G.J., Lefroy R.D. and Lisle L., 1995. Soil carbon fractions based on their degree of oxidation, and the development of a carbon management index for agricultural systems. Crop and Pasture Science, 46, 1459-1466.

Bonachela J.A., Pringle R.M., Sheffer E., Coverdale T.C., Guyton J.A., Caylor K.K., Levin S.A. and Tarnita C.E., 2015. Termite mounds can increase the robustness of dryland ecosystems to climatic change. Science, Vol. 347 , No. 6222, 06.02.2015, p. 651-655.

Booker K., Huntsinger L., Bartolome J.W., Sayre N.F. and Stewart W., 2013. What can ecological science tell us about opportunities for carbon sequestration on arid rangelands in the United States? Global Environmental Change Vol 23 (1), February 2013, Pages 240-251. https://doi.org/10.1016/j.gloenvcha.2012.10.001

Boucher D. and Ferretti-Gallon K., 2015. Halfway There? What the Land Sector Can Contribute to Closing the Emissions Gap. Union of Concerned Scientists.

Brady M.V., Hedlund K., Cong R-G., Hemerik L., Hotes S., Machado S., Mattsson L., Schulz E. and Thomsen I.K., 2015. Valuing supporting soil ecosystem services in agriculture: a Natural Capital Approach. Agronomy Journal, 107, 1809-1821. https://doi.org/10.2134/agronj14.0597

Brady N. and Weil R., 1999. The nature and properties of soil 12th ed. Prentice-Hall Inc. Upper Saddle River, New Jersey. 
Cardinael R., Chevallier T., Cambou A., Beral C., Barthes B.G., Dupraz C., Durand C., Kouakoua E. and Chenu C., 2016. Increase of soil organic carbon stock under agroforestry: a survey of different sites in France. Presentation at Third European Agroforestry Conference, 23-25 May 2016, Montpellier

Comerford N.B., Franzluebbers A.J., Stromberger M.E., Morris L., Markewitz D. and Moore R., 2013. Assessment and evaluation of soil ecosystem services. Soil Horizons, 54.

Conant R.T., Six J. and Paustian K., 2003. Land use effects on soil carbon fractions in the southeastern United States. Management-intensive versus extensive grazing. Biol Fertil Soils (2003) 38:386-392

Cooper P.J., Cappiello S., Vermeulen S., Campbell B.M., Zougmoré R.B. and Kinyangi J., 2013. Large-scale implementation of adaptation and mitigation actions in agriculture.

Dang Y., Ren W., Tao B., Chen G., Lu C., et al., 2014. Climate and Land Use Controls on Soil Organic Carbon in the Loess Plateau Region of China. PLOS ONE 9(5): e95548. https://doi.org/10.1371/journal.pone.0095548

Davies J., Poulsen L., Schulte-Herbrüggen B., Mackinnon K., Crawhall N., Henwood W.D., Dudley N., Smith J. and Gudka M., 2012. Conserving Dryland Biodiversity. IUCN, Nairobi, xii +84p

Davies J., Niamir-Fuller M., Kerven C. and Bauer K., 2010. Extensive Livestock Production in Transition: The Future of Sustainable Pastoralism. In: Steinfeld et al. (eds.), Livestock in a Changing Landscape, Volume 1: Drivers, consequences and responses. Island Press.

Davies J., Ogali C., Laban P., Metternicht G., 2015. Homing in on the Range: Enabling Investments for Sustainable Land Management. Technical Brief 29/01/2015. IUCN Global Drylands Initiative and the IUCN Commission on Ecosystem Management

Diaz-Zorita M., Duarte G.A. and Grove J.H., 2002. A review of non-till systems and soil management for sustainable crop production in the subhumid and semiarid Pampas of Argentina. Soil and Tillage Research 65, 1-18. https://doi. org/10.1016/S0167-1987(01)00274-4

Dumanski J., Peiretti R., Benetis J., McGarry D. and Pieri C., 2006. The paradigm of conservation tillage. Proc. World Assoc. Soil and Water Conserv., P1: 58-64.

ELD INITIATIVE, 2015. The value of land: Prosperous lands and positive rewards through sustainable land management. The Economics of Land Degradation, Bonn, Germany.

Emerson W., 1995. Water-retention, organic-C and soil texture. Soil Research, 33, 241-251. https://doi.org/10.1071/ SR9950241

FAO, 2007. State of the World's forests 2007. Food and Agricultural Organization of the United Nations, Rome.

FAO, 2009. How to Feed the World in 2050. Issues Brief for the High-Level Expert Forum, Rome 12-13 October 2009. Food and Agricultural Organisation of the United Nations, Rome. http://www.fao.org/wsfs/forum2050/wsfsbackground-documents/issues-briefs/en/

FAO, 2017. Soil Organic Carbon: the hidden potential. Food and Agriculture Organisation of the United Nations, Rome.

Farage P., Pretty J. and Ball A., 2003. Biophysical Aspects of Carbon Sequestration in Drylands. University of Essex.

Ferrenberg S., Reed S.C. and Belnap J., 2015. Climate change and physical disturbance cause similar community shifts in biological soil crusts. Proceedings of the National Academy of Sciences, USA 112: 12116-12121

Ferrenberg S., Tucker C. and Reed S., 2017. Biological soil crusts: Diminutive communities of potential global importance. Frontiers in Ecology and the Environment 15(3). https://doi.org/10.1002/fee.1469

Gifford R.M., Cheney N.P., Nobel J.C., Russell J.S., Wellington A.B. and Zammit C., 1992. Australian Land Use, primary production of vegetation and carbon pools in relation to atmospheric carbon dioxide levels. Bureau of Rural Resources Proceeding 14, 151-187.

Gougoulias C., Clark J.M. and Shaw L.J., 2014. The role of soil microbes in the global carbon cycle: tracking the belowground microbial processing of plant-derived carbon for manipulating carbon dynamics in agricultural systems. Journal of the Science of Food and Agriculture, 94, 2362-2371. https://doi.org/10.1002/jsfa.6577

Havlicek E. and Mitchell E.A., 2014. Soils Supporting Biodiversity. Interactions in Soil: Promoting Plant Growth. Springer.

INTERGOVERNMENTAL TECHNICAL PANEL ON SOIL, 2015. Status of the World's Soil Resources. Rome, Italy.

Jenkinson D.S., Harris H.C., Ryan J., Mcneil A.M., Pilbeam C.J. and Coleman K., 1999. Organic matter turnover in calcareous soil from Syria under a two-course cereal rotation. Soil Biology and Biochemistry 31, 687-693. https://doi.org/10.1016/S0038-0717(98)00157-6

Jónsson J.Ö.G. and Davíđsdóttir B., 2016. Classification and valuation of soil ecosystem services. Agricultural Systems, 145, 24-38. https://doi.org/10.1016/j.agsy.2016.02.010

Kibblewhite M., Chambers B. and Goulding K., 2016. How good is the evidence to support investment in soil protection? Soil Use and Management, 32, 172-182. https://doi.org/10.1111/sum.12236 
Kirk G. and Bellamy P., 2010. Analysis of changes in organic carbon in mineral soils across England and Wales using a simple single-pool model. European journal of soil science, 61, 406-411. https://doi.org/10.1111/j.13652389.2010.01242.x

Koch A., Mcbratney A., Adams M., Field D., Hill R., Crawford J., Minasny B., Lal R., Abbott L. and O'Donnell A., 2013. Soil security: solving the global soil crisis. Global Policy, 4, 434-441. https://doi.org/10.1111/1758-5899.12096

Köchy, M., Hiederer R. and Freibauer A., 2015. Global distribution of soil organic carbon-Part 1: Masses and frequency distributions of SOC stocks for the tropics, permafrost regions, wetlands, and the world. Soil, 1, 351-365. https://doi.org/10.5194/soil-1-351-2015

Kpadonou R.A.B., Owiyo T., Barbier B., Denton F., Rutabingwa F. and Kiema A., 2017. Advancing climate-smart-agriculture in developing drylands: Joint analysis of the adoption of multiple on-farm soil and water conservation technologies in West African Sahel. Land Use Policy, 61, 196-207. https://doi.org/10.1016/j.landusepol.2016.10.050

Kumar P., 2010. The economics of ecosystems and biodiversity: ecological and economic foundations, UNEP/Earthprint.

Laban/IUCN-MoA Jordan, 2015. Sustainably Investing in the Jordan Rangelands; a study on rangeland investment options commissioned by IUCN and the Jordan MoA. IUCN ROWA, Amman

Lal R., 2006. Enhancing crop yields in the developing countries through restoration of the soil organic carbon pool in agricultural lands. Land Degradation and Development, 17, 197-209. https://doi.org/10.1002/ldr.696

Lal R., 2008. Soil health and carbon management. Food and Energy Security. Volume 5, Issue 4

Li J., Luo J., Shi Y., Houlbrooke D., Wang L., Lindsey S. and Li Y., 2015. Nitrogen gaseous emissions from farm effluent application to pastures and mitigation measures to reduce the emissions: a review, New Zealand Journal of Agricultural Research. https://doi.org/10.1080/00288233.2015.1028651

Martin-Piera F. and Lobo J.M., 1995. Diversity and ecological role of dung beetles in Iberian grassland biomes. In: Farming on the edge: the nature of traditional farmland in Europe, ed. by. DJ. McCracken, EM. Bignal and SE. Wenlock, 147-153. Peterborough, Joint Nature. Conservation Committee.

McBratney A., Field D.J. and Koch A., 2014. The dimensions of soil security. Geoderma, 213, 203-213. https://doi. org/10.1016/j.geoderma.2013.08.013

McGahey D., Davies J., Hagelberg N. and Ouedraogo R., 2014. Pastoralism and the Green Economy - a natural nexus? Nairobi: IUCN and UNEP. $x+58 p$

Metternicht G., 2018. Land Use and Spatial Planning Enabling Sustainable Management of Land Resources. Springer Briefs in Earth Sciences. 116 pages. Springer Nature: Switzerland.

Milne E., Banwart S.A., Noellemeyer E., Abson D.J., Ballabio C., Bampa F., Bationo A., Batjes N.H., Bernoux M. and Bhattacharyya T., 2015. Soil carbon, multiple benefits. Environmental Development, 13, 33-38. https://doi. org/10.1016/j.envdev.2014.11.005

Nair P.K.R., Kumar B.M. and Nair V., 2009. Agroforestry as a strategy for carbon sequestration. Journal of Plant Nutrition and Soil Science 172(1):10 - 23. https://doi.org/10.1002/jpln.200800030

Nellemann C., 2009. The environmental food crisis: the environment's role in averting future food crises: a UNEP rapid response assessment, UNEP/Earthprint.

NEW CLIMATE ECONOMY, 2014. Better growth, better climate. The New Climate Economy Report, The Global Commission on the Economy and Climate.

Orgiazzi A., Bardgett R.D., Barrios E., Behan-Pelletier V., Briones M.J., Chotte J-L., De Deyn G.B., Eggleton P., Fierer N. and Fraser T., 2016. Global soil biodiversity atlas. Publications Office of the European Union.

O’Rourke S.M., Angers D.A., Holden N.M. and McBratney A.B., 2015. Soil organic carbon across scales. Global change biology, 21, 3561-3574. https://doi.org/10.1111/gcb.12959

Ospina C., 2017. Climate and economic benefits of agroforestry systems. The Climate Institute, Washington

Petersen E. and Hoyle F., 2016. Estimating the economic value of soil organic carbon for grains cropping systems in Western Australia. Soil Research, 54, 383-396. https://doi.org/10.1071/SR15101

Pribyl D.W., 2010. A critical review of the conventional SOC to SOM conversion factor. Geoderma, 156, 75-83. https://doi. org/10.1016/j.geoderma.2010.02.003

Rochecouste J-F., Dargusch P., Cameron D. and Smith C., 2015. An analysis of the socio-economic factors influencing the adoption of conservation agriculture as a climate change mitigation activity in Australian dryland grain production. Agricultural Systems, 135, 20-30. https://doi.org/10.1016/j.agsy.2014.12.002

Rockstrom J., Steffen W., Noone K., Persson Å., Stuart III Chapin F., Lambin E., et al., 2009. Planetary Boundaries: Exploring the Safe Operating Space for Humanity. Resilience Alliance. Ecology and Society 14(2): 32. [online] URL: http://www.ecologyandsociety.org/vol14/iss2/art32/ 
Safriel U., Adeel Z., Niemeijer D., Puigdefabregas J., White R., Lal R., Winslow M., Ziedler J., Prince S., Archner E. and King C., 2005. Dryland systems. In: Hassan, R Scholes, R.J., Ash, N. (Eds.), Ecosystems Human Well-Being. Findings of the Conditions Trends Working Group of the Millennium Ecosystem Assessment, vol. 1. Island Press, Washington D.C., U.S.A, pp. 623-662.).

Scharlemann J.P., Tanner E.V., Hiederer R. and Kapos V., 2014. Global soil carbon: understanding and managing the largest terrestrial carbon pool. Carbon Management, 5, 81-91. https://doi.org/10.4155/cmt.13.77

Sidibe Y., Myint M. and Westerberg V., 2014. An economic valuation of agroforestry and land restoration in the Kelka Forest, Mali. Assessing the socio-economic and environmental dimensions of land degradation. Report for the Economics of Land Degradation Initiative. Nairobi, Kenya.

Silveira M.C.T., da Silva S.C., De Souza Junior S.J., Martins Barbero L., Santos Rodrigues C., Limão V.A., da Silva Pena K. and Do Nascimento Junior D., 2013. Herbage accumulation and grazing losses on Mulato grass subjected to strategies of Márcia Cristina Teixeira da Silveira. Scientia Agricola 704:242-249. https://doi.org/10.1590/ S0103-90162013000400004

Six J., Conant R., Paul E.A. and Paustian K., 2002. Stabilization mechanisms of soil organic matter: implications for C-saturation of soils. Plant and soil, 241, 155-176.

Smith P., 2005. An overview of the permanence of soil organic carbon stocks: influence of direct human-induced, indirect and natural effects. European Journal of Soil Science, October 2005, 56, 673-680

Smith P., 2014. Do grasslands act as a perpetual sink for carbon? Global change biology, 20, 2708-2711. https://doi. org/10.1111/gcb.12561

Smith P., Haberl H., Popp A., Erb K.H., Lauk C., Harper R., Tubiello F.N., Siqueira Pinto A., Jafari M. and Sohi S., 2013. How much land-based greenhouse gas mitigation can be achieved without compromising food security and environmental goals? Global Change Biology, 19, 2285-2302. https://doi.org/10.1111/gcb.12160

Sudgen A., Stone R. and Ash C., 2004. Soils-The Final Frontier. Science, 304, 1613.

Teague W.R., Dowhowera S.L., Baker S.A., Haile N, DELAUNE PB and CONOVER DM, 2011. Grazing management impacts on vegetation, soil biota and soil chemical, physical and hydrological properties in tall grass prairie. Agriculture, Ecosystems and Environment, Vol 141, Issues 3-4, Pages 310-322. https://doi.org/10.1016/j. agee.2011.03.009

UNCCD SPI, 2017 Policy Brief No. 3: Sustainable Land Management Solutions. http://www2.unccd.int/sites/default/files/ documents/2017-09/Policy_brief_ENG.pdf

UNESCO, 2012. The United Nations World Water Development Report 4: Managing Water under Uncertainty and Risk. Paris, UNESCO.

UNEP, 2011. Towards a Green Economy: Pathways to Sustainable Development and Poverty Eradication. United Nations Environment Programme, Nairobi.

van Der Putten W.H., Anderson J., Bardgett R., Behan-Pelletier V., Bignell D., Brown G., Brown V., Brussaard L., Hunt H. and Ineson P., 2004. The sustainable delivery of goods and services provided by soil biota. Sustaining biodiversity and ecosystem services in soils and sediments: $15-43$.

van Der Wal A. and De Boer W., 2017. Dinner in the dark: Illuminating drivers of soil organic matter decomposition. Soil Biology and Biochemistry, 105, 45-48. https://doi.org/10.1016/j.soilbio.2016.11.006

Vignola R., Harvey C.A., Bautista-Solis P., Avelino J., Rapidel B., Donatti C. and Martinez R., 2015. Ecosystem-based adaptation for smallholder farmers: Definitions, opportunities and constraints, In Agriculture, Ecosystems and Environment, Volume 211, 2015, Pages 126-132, ISSN 0167-8809

Wang S., Wilkes A., Zhang Z., Chang X., Lang R., Wang Y. and Niu H., 2011. Management and land use change effects on soil carbon in northern China's grasslands: a synthesis, Agriculture, Ecosystems and Environment 142 (2011) 329- 340. https://doi.org/10.1016/j.agee.2011.06.002

Watson L., 2010. Portugal gives green light to pasture carbon farming as a recognised offset. Australian Farm Journal, 44-47.

Weber J-L., 2007. Accounting for soil in the SEEA. European Environment Agency, Rome.

Weissert L., Salmond J. and Schwendenmann L., 2016. Variability of soil organic carbon stocks and soil CO2 efflux across urban land use and soil cover types. Geoderma, 271, 80-90. https://doi.org/10.1016/j.geoderma.2016.02.014

Zomer R.J., Trabucco A., Coe R. and Place F., 2009. Trees on farm: analysis of global extent and geographical patterns of agroforestry. ICRAF Working Paper. Nairobi: World Agroforestry Centre (ICRAF). 


\section{Notes de fin de texte}

1. Sudgen et al., 2004, Weber, 2007; Jónsson and Davíðsdóttir, 2016; Brady and Weil, 1999

2. Orgiazzi et al., 2016

3. Ferrenberg et al., 2015; Ferrenberg et al., 2017

4. Bernoux and Chevallier, 2014; Pribyl, 2010; FAO, 2017; Kibblewhite et al., 2016

5. Bonachela et al., 2015; Marint-Piera and Lobo, 1995; Orgiazzi et al., 2016.

6. Belnap and Lange, 2003

7. Brady and Weil, 1999

8. Van der Putten et al., 2004

9. Amundson et al., 2015

10. Emerson, 1995

11. Havlicek and Mitchell, 2014

12. Bernoux and Chevallier, 2014

13. Brady et al., 2015

14. Available from www.iucn.org/drylands:

- Land Degradation Neutrality: implications and opportunities for conservation. https://www.iucn.org/ sites/dev/files/content/documents/tech_brief_land_degradation_neutrality_revised_2017_2.pdf

- Homing in on the Range: Enabling Investments for Sustainable Land Management. http://cmsdata. iucn.org/downloads/technical_brief__investing_in_slm_2.pdf

15. Banwart et al., 2014; Milne et al., 2015; Baveye et al., 2016; van der Wal and de Boer, 2017; Bernoux and Chevallier, 2014; FAO, 2017, Havlicek and Mitchell, 2014; Brady et al., 2015

16. ELD Initiative, 2015

17. van der Putten et al., 2004

18. Boucher and Ferretti-Gallon, 2015

19. Bai et al., 2008

20. FAO, 2009; UNESCO, 2012; Nellemann, 2009

21. New Climate Economy, 2014

22. ELD Initiative, 2015

23. Scharlemann et al., 2014; Batjes, 2016

24. Weissert et al., 2016; Batjes, 2016

25. Gougoulias et al., 2014; Köchy et al., 2015

26. O'Rourke et al., 2015

27. Blair et al., 1995; FAO, 2017

28. Petersen and Hoyle, 2016

29. Kibblewhite et al., 2016; Kirk and Bellamy, 2010; Smith, 2014

30. Davies et al., 2012

31. Bastin et al., 2017

32. http://www.unccd.int/Lists/SiteDocumentLibrary/WDCD/DLDD\%20Facts.pdf

33. http://www.fao.org/fileadmin/user_upload/newsroom/docs/i0372e01.pdf

34. Davies et al, 2012

35. Safriel et al, 2005

36. Davies et al., 2015

37. Safriel et al, 2005

38. Bernoux and Chevallier, 2014; Lal, 2008

39. Gifford et al., 1992

40. Smith 2005

41. Six et al., 2002; Beare et al., 2014

42. Álvaro-Fuentes et al, 2007

43. Laban/IUCN-MoA Jordan, 2015

44. Diaz-Zorita et al., 2002; Jenkinson et al., 1999.

45. Farage et al., 2003

46. FAO, 2007

47. Batjes 2004

48. FAO, 2009

49. UNEP, 2011; Intergovernmental Technical Panel on Soil, 2015; FAO, 2017

50. McBratney et al.,2014

51. McBratney et al., 2014; Koch et al., 2013; Bernoux and Chevallier, 2014

52. Bernoux and Chevallier, 2014

53. FAO, 2017

54. Dumanski et al., 2006

55. Davies et al., 2010; McGahey et al., 2014

56. http://www.fao.org/forestry/agroforestry/80338/en/

57. Vignola et al, 2015

58. Laban/IUCN-MoA Jordan, 2015

59. Conant et al. 2003 
60. Wang et al. 2011; Silveira et al. 2013; Smith et al. 2013

61. Teague et al. 2011

62. McGahey et al. 2014

63. Li et al. 2013

64. Nair et al., 2009; Zomer et al., 2009

65. Lal, 2006

66. Metternicht, 2018

67. Aerni, P. 2016

68. Jónsson and Davíðsdóttir, 2016; Adhikari and Hartemink, 2016; Comerford et al., 2013; Kumar, 2010; Banwart et al., 2014; Baveye et al., 2016; Milne et al., 2015; Brady et al., 2015

69. A summary of the economic value estimated in the literature for a range of different ecosystem services provided by soil can be found in Cameron et al., 2017

70. Kpadonou et al., 2017

71. Davies et al., 2015; Laban/IUCN-MoA Jordan, 2015

72. UNCCD SPI, 2017

73. Rowarth, 2017; Rockstrom, 2009

74. Sidibe et al., 2014

75. Cardinael, et al, 2016; Ospina, 2017

76. Dang et al., 2014; Cooper et al., 2013; New Climate Economy, 2014

77. Rochecouste et al., 2015; Australian Government, 2012

78. Booker et al 2013

79. https://www.cbd.int/sp/targets/rationale/target-11/

80. Watson, 2010

81. Bishop and Hill, 2014; Laban/IUCN-MoA Jordan, 2015

82. Laban/IUCN-MoA Jordan, 2015

83. Arriagada and Perrings, 2009 



\section{IUCN}

UNION INTERNATIONALE POUR LA CONSERVATION DE LA NATURE

Initiative Mondiale pour les Zones arides Mukoma Road (off Magadi Rd)

P. O. Box 68200 - 00200

NAIROBI, Kenya

Tel: +254 20249 3561/65

+254724256804

+254734768 770

www.iucn.org

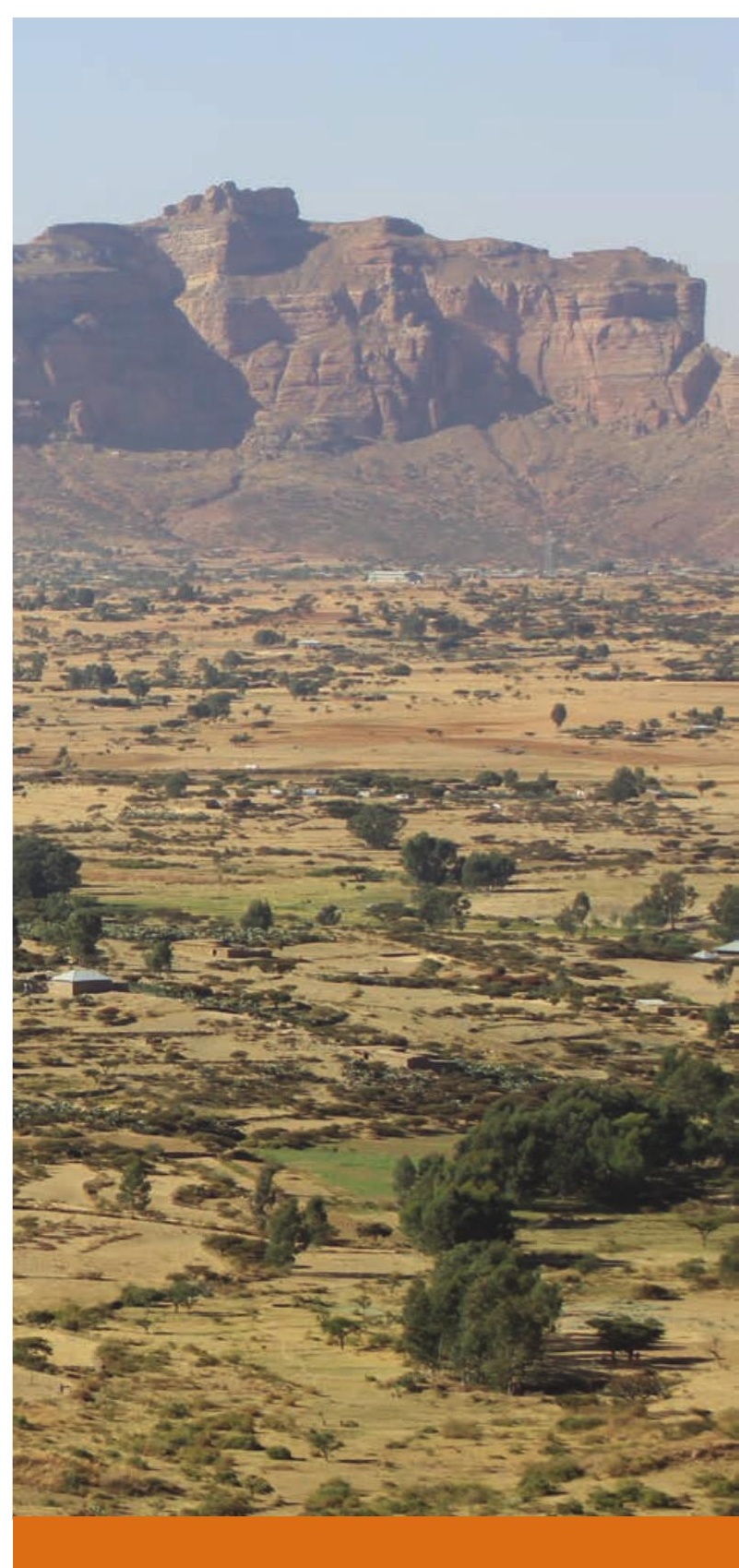

